NASA TECHNICAL NOTE

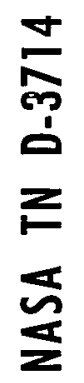

LOAN COPY: RETL AFWL (WLIL-2, KIRTLAND AFB, N MEXX

\title{
A STUDY OF EMBEDMENT AND OTHER METALLURGICAL AND MECHANICAL CHARACTERISTICS OF CROSS-WIRE RESISTANCE WELDS
}

by James $A$. Munford

Goddard Space Flight Center Greenbelt, $M d$.

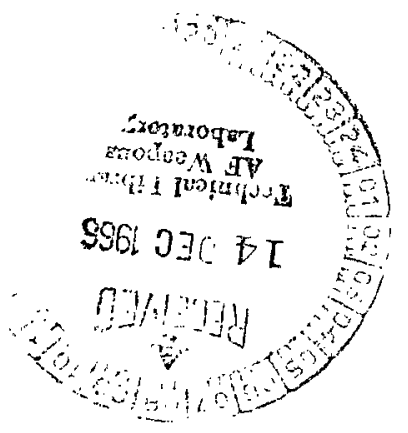

NATIONAL AERONAUTICS AND SPACE ADMINISTRATION - WASHINGTON, D. C. - NOVEMBER 1966 


\section{A STUDY OF EMBEDMENT AND OTHER}

\section{METALLURGICAL AND MECHANICAL CHARACTERISTICS OF CROSS-WIRE RESISTANCE WELDS}

By James A. Munford

Goddard Space Flight Center Greenbelt, $\mathrm{Md}$.

NATIONAL AERONAUTICS AND SPACE ADMINISTRATION

For sale by the Cleoringhouse for Federal Scientific and Technical Information Springfield, Virginia 22151 - Price $\$ 1.00$ 


\begin{abstract}
An investigation was performed to evaluate the crosswire resistance welded joints used in welded electronic assemblies for aerospace applications. Mechanical strength and reproducibility of strength were determined for several alloy combinations by performing single torsion-shear tests in a pneumatically operated pull testing machine. A metallographic investigation was performed in order to determine the type of bonding and the degree of mutual embedment resulting from the welding operation.

Material combinations investigated were Nickel 200 interconnect welded to itself, Kovar, Alloy 180, and Dumet; and Alloy 180 interconnect welded to itself, Kovar, and Dumet. Strength, embedment, and consistency data indicate that an optimum degree of embedment exists for each combination of materials. For the material combinations studied, the optimum embedment was found to be between 10 and 25 percent of the wire diameter.
\end{abstract}


CONTENTS

Abstract. ..................... ii

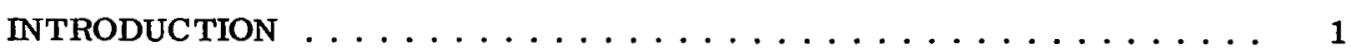

EQUIPMENT ......................... 3

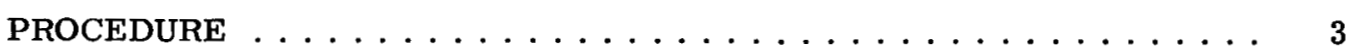

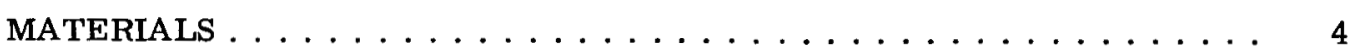

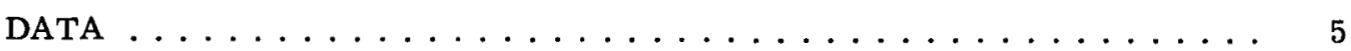

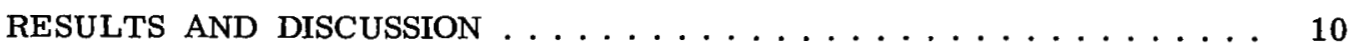

Nickel 200 welded to Nickel $200 \ldots \ldots \ldots \ldots$. . . . . . . . 10

Nickel 200 welded to Dumet. . . . . . . . . . . . . . . . . . . . 12

Nickel 200 Welded to Kovar . . . . . . . . . . . . . . . . . 14

Nickel 200 welded to Alloy $180 \ldots \ldots \ldots \ldots \ldots$

Alloy 180 welded to Alloy $180 \ldots \ldots \ldots \ldots \ldots$

Alloy 180 welded to Dumet . . . . . . . . . . . . . . . 18

Alloy 180 Welded to Kovar . . . . . . . . . . . . . . . . . . . 19

Alloy 180 Welded to Alloy 180 Ribbon $\ldots \ldots \ldots \ldots \ldots \ldots$

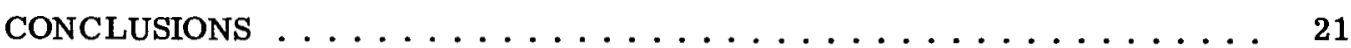

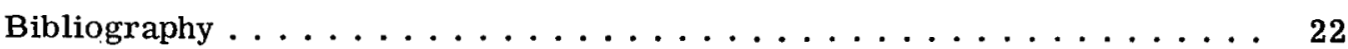




\title{
A STUDY OF EMBEDMENT AND OTHER METALLURGICAL AND MECHANICAL CHARACTERISTICS OF CROSS-WIRE RESISTANCE WELDS
}

\author{
by \\ James A. Munford \\ Goddard Space Flight Center
}

\section{INTRODUCTION}

The advent of space exploration has placed an ever increasing demand upon the electronics industry to provide smaller, lighter, more reliable, and more complex electronic packages. Welded circuitry provides a balance between the weight and physical size of printed circuit assemblies and the high cost and limited power applications of thin film circuitry. The welded joints also exhibit higher mechanical strengths than soldered joints.

The joints may be produced by fusion welding (Figure 1), by recrystallizing the interfacial surfaces (Figure 2), by brazing (Figure 3), or by pressure welding. Evidence of two or more of these mechanis ms may be found in a single joint. Any of these mechanisms can be expected to produce joints having higher mechanical strength properties than soldered joints. Generally, the type of weld joint obtained is dependent upon the welding energy, the electrode force and the melting temperatures and thermal conductivities of the materials being welded. Of the four mechanisms, the fusion nugget has greater reliability and consistency because the cleanliness and the weld settings are not as critical. This does not mean, however, that the formation of a fusion nugget constitutes a satisfactory weld. Many times it indicates that excessive weld energy and insufficient electrode force has been employed, resulting in joints having strength and consistency values that are less than optimum. Strength alone, however, is not the criterion for an optimum weld schedule. The reproducibility of the weld strength may be of

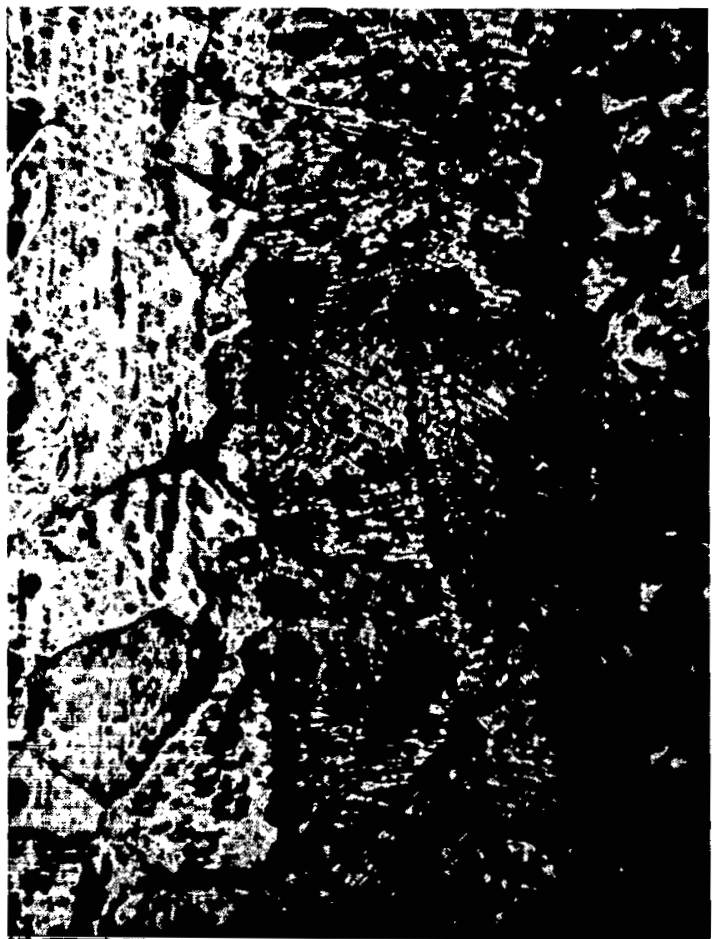

Figure 1-Photomicrograph showing a fusion nugget in a weld joint of Alloy 180 (magnified $500 \mathrm{X})$. 


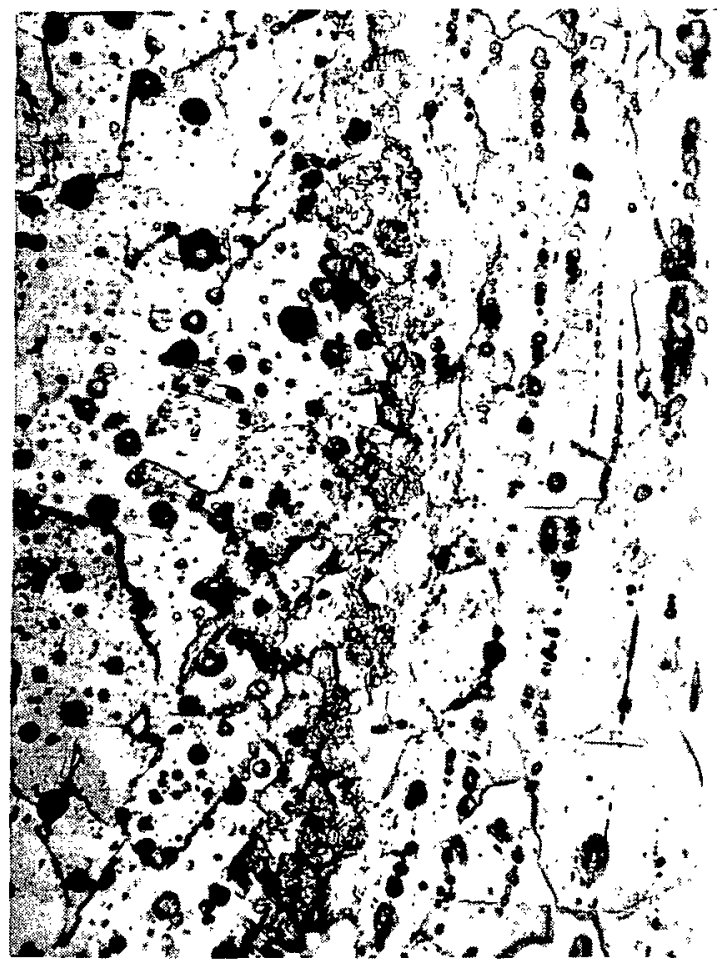

Figure 2-Photomicrograph showing a recrystallized interface in a nickel weld joint (magnified 500X).

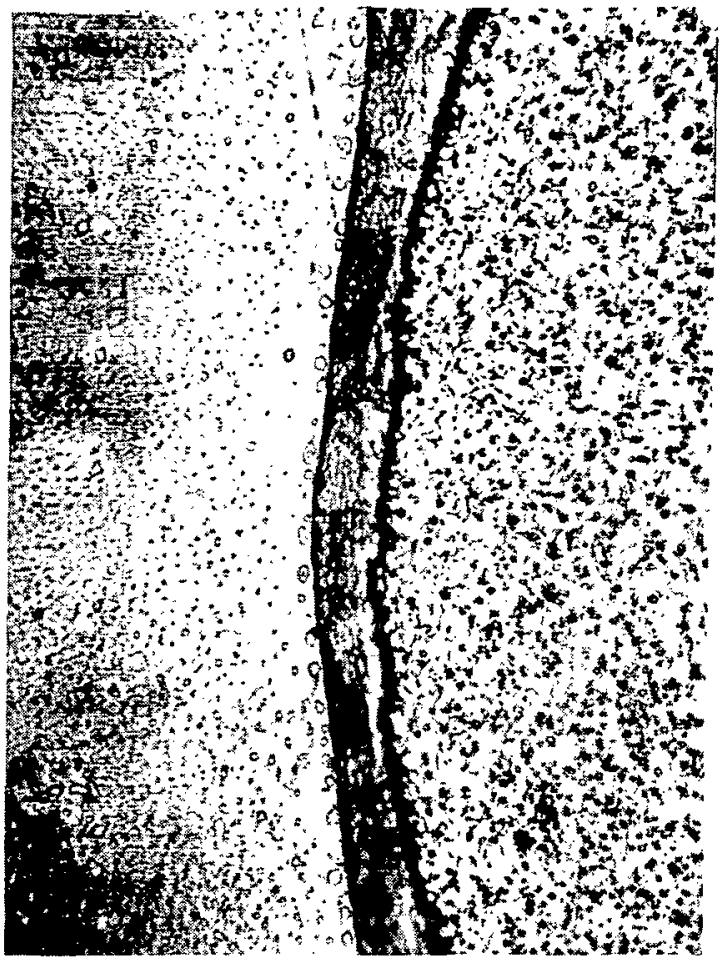

Figure 3-Photomicrograph of a braze joint produced between the copper sheath of Dumet (right) and nickel (magnified 500X).

greater importance in establishing a weld schedule than the tensile-shear strength alone. A few pounds of the average pull strength may well be sacrificed to attain the desired reproducibility.

Claddings, coatings, and platings may also influence the type of bond produced. High melting point coatings such as silver, copper, or gold may remain in the joint interface and produce a brazed joint. Low melting point coatings such as tin, lead, or solder do not affect the type of weld, in most cases, because they are quickly melted and forced out of the joints.

Resistance welded joints are normally evaluated on the basis of their mechanical strength, visual appearance, and metallographic appearance. Since mechanical strength and metallographic appearance cannot be determined on an actual production weld joint, statistical quality control has become the standard method of determining production weld integrity. The combination of electrodes, electrode force, and energy input used to produce consistently high strength welded joints is usually determined by an empirical method. One such method involves systematically varying the electrode force and energy input to select the settings that produce a combination of the highest strength and the lowest standard deviation. Strength and reproducibility data alone, however, are not sufficient to establish a production weld schedule. Mechanical tests should be supplemented with visual and metallurgical examinations. Although appearance alone has been used by some fabricators as a measure of weld integrity, this method cannot be expected to yield highly reliable welded joints. 
The criteria used for visual examination generally involves only a search for external defects, misaligned or off-center welds, and excessive or insufficient embedment. The determination of embedment is best accomplished by a metallographic examination, however an estimate can be made visually under a magnification of 10 to 15 diameters. Embedment values may also be determined in some instances by measuring the thickness of welded joints with micrometers. When space permits, this method may offer a non-destructive method of monitoring joint quality when the strength-variation-embedment relationships have been established for an alloy and size combination. Although one specification (Bureau of Naval Weapons OD 11889B) requires that embedment be greater than 5 percent and not more than 75 percent, regardless of the alloy or the size of the lead materials, it is found that for a particular combination of materials, there is an optimum degree of embedment corresponding to the optimum weld schedule; that is, one producing welds having high strength and a minimum standard deviation.

\section{EQUIPMENT}

The welder (Figure 4) used to develop the data in this report is composed of a power supply, a junction box, and a weld head.

The power supply is of the capacitive discharge type and is capable of producing up to 40 watt-seconds of energy. The weld pulse is approximately $1-1 / 2$ milliseconds through a normal impedance weld joint, and can be repeated every two seconds at maximum power. Energy storage is variable from 0.4 to 40 watt-seconds.

A wide range pincer weld head having two fixed-angle electrode configurations was used in conjunction with the power supply. This head provides spring controlled variable

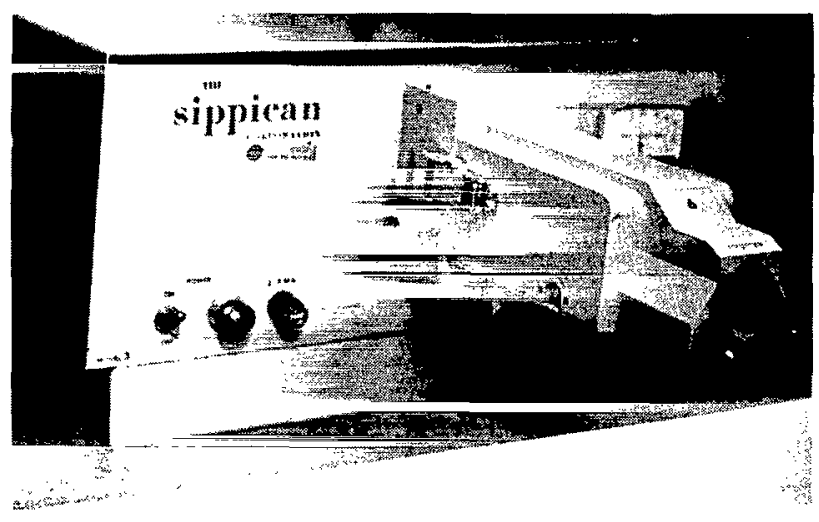

Figure 4-Capacitive discharge welder with pincer head. electrode forces from $1 / 4$ to 10 pounds and a low inertia of 0.5 ounce. The electrode.tip angle can be set at 60 degrees for normal production or 36 degrees for applications where workspace is limited.

\section{PROCEDURE}

Over eight thousand weld samples were made with 0.020 inch diameter wire at various electrode force and weld energy settings. Sixty welds were made at each two-pound force increment and each two-watt-second energy increment. Fifty welds made at each setting were tensile-shear tested (Figure 5) on a pneumatically operated mechanical tester (Figure 6). From this data, the average strength and standard deviation was computed. 


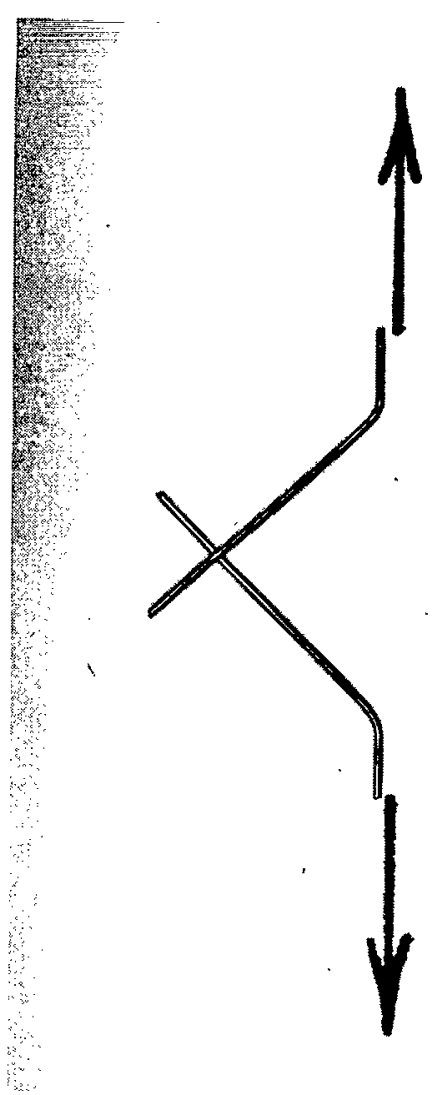

Figure 5-Typical cross-wire weld joint showing direction of loading for tensile-shear tests.

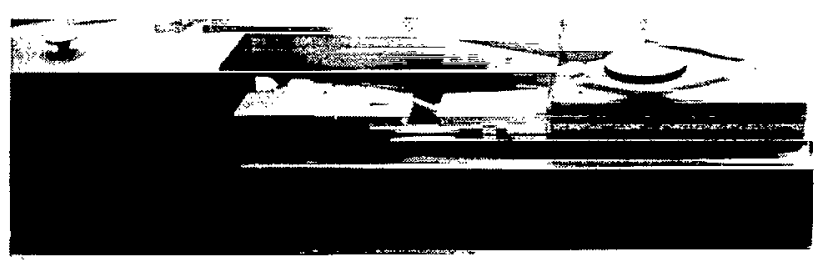

Figure 6-Pneumatic pull tester for determining tensile-shear strength.

The remaining samples were mounted in plastic, ground on successively finer grades of silicon carbide paper, then polished metallurgically using a diamond abrasive followed by aluminum oxide. The amount of embedment of one wire into the other was measured microscopically using a filar eyepiece. The type of bonding and the role of platings or coatings were determined by microscopic examination on a research metallograph. Average pull strength, standard deviation, and welding energy at constant electrode forces were then plotted against embedment, and the optimum degree of embedment was determined for each combination of materials.

One additional combination of materials $(0.020 \mathrm{inch}$ diameter wire of Alloy 180 welded to 0.012 -inch by $0.022-$ inch ribbon of Alloy 180) was welded at various force and energy settings to determine if micrometer meas-

urements of embedment could be effective in providing a non-destructive method of evaluating weld joint quality. The thicknesses of the weld joints were measured with barrel micrometers and the embedments calculated. These samples were then tensile-shear tested to determine the average strength and standard deviation at each machine setting.

\section{MATERIALS}

The combinations of materials selected for this investigation represent those normally encountered in electronic module production. Both Nickel 200 and Alloy 180 are commonly used as interconnect materials, and preferred component leads are usually Nickel 200, Kovar, or Dumet. Alloy 180 is also available as a lead material on components from some suppliers.

Nickel 200 is a commercially pure nickel widely used as an interconnecting medium in the fabrication of welded electronic modules. Its chemical composition is controlled to a minimum of 99.00 percent nickel (+ cobalt). This material has excellent weldability over a wide range of welding variables in addition to high electrical conductivity and inherent corrosion resistance. 
Alloy 180 is a copper-nickel alloy consisting of 78 percent copper and 22 percent nickel. This material is frequently used as the interconnecting medium on electronic modules where a nonmagnetic requirement exists. It also exhibits good weldability, high electrical conductivity, and good corrosion resistance.

Kovar is an iron base alloy consisting of 54 percent iron, 29 percent nickel, and 17 percent cobalt. This alloy is widely used for leads on transistors and other components employing hard glass seals because of its compatible coefficient of expansion. Since Kovar is not inherently corrosion resistant, it is usually supplied with gold plating or with a solder or tin coating.

Diodes, capacitors, and resistors having soft glass seals are often supplied with Dumet leads, an alloy of 42 percent nickel and 58 percent iron with a copper sheath. The coefficient of expansion of this alloy is controlled to match many soft glasses used in hermetically sealed components.

Numerous other metals and alloys are occasionally found as lead materials on components intended for use in welded electronic modules. However, in an effort to limit the number of lead materials and weld schedules, the Department of Defense issued Military Standard 1276, "Weldable Leads for Electronic Component Parts," which specifies the use of Dumet, Kovar, nickel, and copper for component leads unless other materials are specified for special applications. This standard also limits the chemical composition of the base materials, the thicknesses and chemical compositions of platings and coatings, and establishes the sizes of wire and flat ribbon to be used as lead materials on electronic components intended for welding.

\section{DATA}

The data contained in Tables 1 through 8 represent findings from more than eight thousand cross-wire weld samples. Strength values given for each welder setting represent an average of 50 tensile-shear tests and embedment values represent the average of 10 microscopic measurements.

Table 1

0.020 Inch Diameter Nickel 200 Welded to 0.020 Inch Diameter Nickel 200.

\begin{tabular}{|c|c|c|c|c|c|}
\hline $\begin{array}{l}\text { Electrode } \\
\text { Force } \\
\text { (lbs.) }\end{array}$ & $\begin{array}{c}\text { Welding } \\
\text { Energy } \\
\text { (watt-sec.) }\end{array}$ & $\begin{array}{c}\text { Average } \\
\text { Strength } \\
\text { (lbs.) }\end{array}$ & $\begin{array}{c}\text { Standard } \\
\text { Deviation }\end{array}$ & $\begin{array}{c}\text { Average } \\
\text { Embedment } \\
\text { (percent wire diam.) }\end{array}$ & Bond Type \\
\hline 3 & 2 & 6.1 & 1.14 & 5.5 & Recrystallized Interface \\
\hline 3 & 4 & 12.1 & 0.40 & 11.2 & Recrystallized Interface \\
\hline 3 & 6 & 13.8 & 0.56 & 14.5 & R. I. and Fusion Nugget \\
\hline 3 & 8 & 15.4 & 0.76 & 14.7 & R. I. and Fusion Nugget \\
\hline 3 & 10 & 17.0 & 1.10 & 20.7 & R. I. and Fusion Nugget \\
\hline 3 & 12 & 16.7 & 0.63 & 17.7 & Fusion Nugget \\
\hline 3 & 14 & 17.6 & 0.82 & 18.5 & Recrystallized Interface \\
\hline 5 & 2 & 0.7 & 0.54 & 3.4 & Recrystallized Interface \\
\hline 5 & 4 & 10.4 & 0.92 & 6.3 & Recrystallized Interface \\
\hline
\end{tabular}


Table 1 (continued)

\begin{tabular}{|c|c|c|}
\hline $\begin{array}{c}\text { Electrode } \\
\text { Force } \\
\text { (lbs.) }\end{array}$ & $\begin{array}{c}\text { Welding } \\
\text { Energy } \\
\text { (watt-sec.) }\end{array}$ & $\begin{array}{c}\text { Average } \\
\text { Strength } \\
\text { (lbs.) }\end{array}$ \\
\hline 5 & 6 & 13.6 \\
5 & 8 & 14.5 \\
5 & 10 & 15.5 \\
5 & 12 & 16.7 \\
5 & 14 & 17.9 \\
7 & 4 & 8.5 \\
7 & 6 & 14.3 \\
7 & 8 & 15.9 \\
7 & 10 & 16.9 \\
7 & 12 & 18.3 \\
7 & 14 & 18.5 \\
7 & 16 & 19.8 \\
9 & 4 & 1.0 \\
9 & 6 & 13.5 \\
9 & 8 & 16.0 \\
9 & 10 & 17.3 \\
9 & 12 & 18.6 \\
9 & 14 & 20.0 \\
9 & 16 & 19.7 \\
\hline
\end{tabular}

\begin{tabular}{l|c|} 
Standard & $\begin{array}{c}\text { Average } \\
\text { Deviation }\end{array}$ \\
0.59 & $\begin{array}{c}\text { Embedment } \\
\text { (percent wire diam.) }\end{array}$ \\
0.66 & 8.2 \\
0.69 & 9.8 \\
0.80 & 15.5 \\
1.04 & 15.4 \\
0.98 & 16.2 \\
0.56 & 3.4 \\
0.88 & 5.5 \\
0.71 & 9.4 \\
0.92 & 12.0 \\
1.07 & 15.0 \\
0.86 & 17.0 \\
0.76 & 18.0 \\
1.34 & 4.3 \\
1.56 & 6.5 \\
1.34 & 8.5 \\
0.73 & 10.0 \\
0.92 & 19.0 \\
1.49 & 15.2 \\
& 16.4
\end{tabular}

Bond Type

Recrystallized Interface Recrystallized Interface Recrystallized Interface Recrystallized Interface Recrystallized Interface Recrystallized Interface Recrystallized Interface Recrystallized Interface Recrystallized Interface Recrystallized Interface Recrystallized Interface Recrystallized Interface Recrystallized Interface Recrystallized Interface Recrystallized Interface Recrystallized Interface Recrystallized Interface Recrystallized Interface Recrystallized Interface

Table 2

0.020 Inch Diameter Nickel 200 Welded to 0.020 Inch Diameter Dumet.

\begin{tabular}{|c|c|c|}
\hline $\begin{array}{c}\text { Electrode } \\
\text { Force } \\
\text { (lbs.) }\end{array}$ & $\begin{array}{c}\text { Welding } \\
\text { Energy } \\
\text { (watt-sec.) }\end{array}$ & $\begin{array}{r}\text { Average } \\
\text { Strength } \\
\text { (lbs.) }\end{array}$ \\
\hline 3 & 2 & 0.4 \\
3 & 4 & 10.3 \\
3 & 6 & 13.0 \\
3 & 8 & 13.4 \\
3 & 10 & 12.4 \\
5 & 2 & 0.3 \\
5 & 4 & 11.8 \\
5 & 6 & 14.6 \\
5 & 8 & 13.7 \\
5 & 10 & 12.2 \\
5 & 12 & 11.5 \\
7 & 2 & 0.2 \\
7 & 4 & 12.5 \\
7 & 6 & 15.9 \\
7 & 8 & 16.7 \\
7 & 10 & 13.8 \\
7 & 12 & 12.0 \\
9 & 2 & 0.2 \\
9 & 4 & 10.0 \\
9 & 6 & 13.8 \\
9 & 12 & 14.5 \\
9 & & 12.9 \\
9 & 12.2 \\
\hline
\end{tabular}

\begin{tabular}{|c|} 
Standard \\
Deviation \\
\\
0.18 \\
0.90 \\
0.76 \\
1.14 \\
3.29 \\
0.21 \\
1.37 \\
1.04 \\
1.48 \\
1.37 \\
0.58 \\
0.10 \\
1.24 \\
1.07 \\
1.85 \\
2.57 \\
0.52 \\
0.03 \\
1.50 \\
0.86 \\
1.24 \\
1.69 \\
1.92 \\
\end{tabular}

Avera
Embedm
(percent wir
4.4
12.5
15.3
21.2
27.0
7.6
13.8
16.5
21.2
27.6
32.0
3.9
10.0
17.2
18.7
28.9
31.7
0.5
9.8
18.0
21.5
29.9
33.0

Bond Type

Copper Braze

Recrystallized Interface Recrystallized Interface Recrystallized Interface R. I. and Fusion Nugget Copper Braze

Recrystallized Interface Recrystallized Interface Recrystallized Interface Recrystallized Interface R. I. and Fusion Nugget Copper Braze

Recrystallized Interface Recrystallized Interface Recrystallized Interface Recrystallized Interface Recrystallized Interface Copper Braze

Recrystallized Interface Recrystallized Interface Recrystallized Interface Recrystallized Interface Recrystallized Interface 
Table 3

0.020 Inch Diameter Nickel 200 Welded to 0.020 Inch Diameter Kovar (Gold Plated).

\begin{tabular}{|c|c|c|c|l|}
$\begin{array}{c}\text { Welding } \\
\text { Energy } \\
\text { (watt-sec.) }\end{array}$ & $\begin{array}{c}\text { Average } \\
\text { Strength } \\
\text { (lbs.) }\end{array}$ & $\begin{array}{c}\text { Standard } \\
\text { Deviation }\end{array}$ & $\begin{array}{c}\text { Average } \\
\text { Embedment } \\
\text { (percent wire diam.) }\end{array}$ & \multicolumn{1}{|c|}{ Bond Type } \\
2 & 8.8 & 1.06 & 4.8 & Recrystallized Interface \\
4 & 12.5 & 0.87 & 13.9 & R. I. and Fusion Nugget \\
6 & 13.6 & 1.28 & 17.7 & Fusion Nugget \\
8 & 8.9 & 5.45 & 20.2 & Fusion Nugget \\
2 & 8.9 & 1.98 & 4.9 & Recrystallized Interface \\
4 & 13.9 & 1.22 & 12.3 & R. I. and Fusion Nugget \\
6 & 12.7 & 2.77 & 23.7 & Fusion Nugget \\
8 & 3.9 & 3.80 & 28.4 & Fusion Nugget \\
2 & 8.5 & 2.73 & 17.9 & Recrystallized Interface \\
4 & 11.2 & 1.52 & 26.2 & Recrystallized Interface \\
6 & 11.1 & 2.22 & 39.4 & Fond Fusion Nugget \\
8 & 6.3 & 3.44 & 7.1 & Fusion Nugget \\
2 & 6.6 & 2.70 & 16.4 & Recrystallized Interface \\
4 & 10.2 & 1.91 & 25.9 & Fusion Nugget \\
6 & 9.9 & 1.41 & 34.6 & Fusion Nugget \\
8 & 7.9 & 2.26 & &
\end{tabular}

Table 4

0.020 Inch Diameter Nickel 200 Welded to 0.020 Inch Diameter Alloy 180.

\begin{tabular}{|c|c|c|c|c|c|}
\hline $\begin{array}{l}\text { Electrode } \\
\text { Force } \\
\text { (lbs.) }\end{array}$ & $\begin{array}{c}\text { Welding } \\
\text { Energy } \\
\text { (watt-sec.) }\end{array}$ & $\begin{array}{c}\text { Average } \\
\text { Strength } \\
\text { (lbs.) }\end{array}$ & $\begin{array}{c}\text { Standard } \\
\text { Deviation }\end{array}$ & $\begin{array}{c}\text { Average } \\
\text { Embedment } \\
\text { (percent wire diam.) }\end{array}$ & Bond Type \\
\hline 3 & 2 & 0.2 & 0.03 & 1.2 & Recrystallized Interface \\
\hline 3 & 4 & 4.0 & 1.19 & 2.5 & Recrystallized Interface \\
\hline 3 & 6 & 8.0 & 0.98 & 3.8 & Recrystallized Interface \\
\hline 3 & 8 & 9.5 & 0.84 & 11.8 & Recrystallized Interface \\
\hline 3 & 10 & 9.4 & 0.69 & 17.4 & Fusion Nugget \\
\hline 3 & 12 & 9.0 & 1.30 & 22.5 & Fusion Nugget \\
\hline 5 & 2 & 0.2 & 0.12 & - & Recrystallized Interface \\
\hline 5 & 4 & 1.5 & 1.47 & 9.1 & Recrystallized Interface \\
\hline 5 & 6 & 8.9 & 0.64 & 12.4 & Recrystallized Interface \\
\hline 5 & 8 & 9.9 & 0.80 & 14.6 & Recrystallized Interface \\
\hline 5 & 10 & 9.1 & 0.88 & 17.5 & Fusion Nugget \\
\hline 5 & 12 & 8.5 & 0.80 & 20.7 & Fusion Nugget \\
\hline 7 & 4 & 0.2 & 0.14 & 6.9 & Recrystallized Interface \\
\hline 7 & 6 & 8.6 & 0.63 & 10.4 & Recrystallized Interface \\
\hline 7 & 8 & 10.5 & 0.99 & 12.3 & Recrystallized Interface \\
\hline 7 & 10 & 9.8 & 0.83 & 18.8 & Fusion Nugget \\
\hline 7 & 12 & 6.3 & 2.09 & 22.1 & Fusion Nugget \\
\hline 9 & 4 & 0.1 & 0.05 & - & Recrystallized Interface \\
\hline 9 & 6 & 2.0 & 1.29 & 11.4 & Recrystallized Interface \\
\hline 9 & 8 & 9.2 & 0.88 & 14.0 & Recrystallized Interface \\
\hline 9 & 10 & 9.4 & 1.74 & 23.2 & Fusion Nugget \\
\hline 9 & 12 & 11.1 & 1.14 & 20.4 & Fusion Nugget \\
\hline
\end{tabular}


Table 5

0.020 Inch Diameter Alloy 180 Welded to 0.020 Inch Diameter Alloy 180.

\begin{tabular}{|c|c|c|c|c|}
\hline $\begin{array}{l}\text { Electrode } \\
\text { Force } \\
\text { (lbs.) }\end{array}$ & $\begin{array}{c}\text { Welding } \\
\text { Energy } \\
\text { (watt-sec.) }\end{array}$ & $\begin{array}{l}\text { Average } \\
\text { Strength } \\
\text { (lbs.) }\end{array}$ & $\begin{array}{c}\text { Standard } \\
\text { Deviation }\end{array}$ & $\begin{array}{c}\text { Average } \\
\text { Embedment } \\
\text { (percent wire diam.) }\end{array}$ \\
\hline 3 & 4 & 8.8 & 0.42 & 10.3 \\
\hline 3 & 6 & 10.8 & 0.44 & 15.8 \\
\hline 3 & 8 & 11.8 & 0.45 & 22.6 \\
\hline 3 & 10 & 11.4 & 0.73 & 27.8 \\
\hline 3 & 12 & 11.5 & 0.86 & 27.2 \\
\hline 5 & 4 & 9.2 & 0.42 & 8.4 \\
\hline 5 & 6 & 11.1 & 0.42 & 12.7 \\
\hline 5 & 8 & 12.1 & 0.39 & 19.0 \\
\hline 5 & 10 & 9.2 & 3.55 & 27.8 \\
\hline 5 & 12 & 4.0 & 4.90 & 32.8 \\
\hline 7 & 8 & 12.7 & 0.22 & 21.6 \\
\hline 7 & 10 & 12.7 & 0.30 & 22.2 \\
\hline 7 & 12 & 7.3 & 4.32 & 28.8 \\
\hline 7 & 14 & 10.1 & 3.12 & 26.6 \\
\hline 7 & 16 & 2.0 & 3.58 & 31.4 \\
\hline 7 & 18 & 4.8 & 4.29 & 28.7 \\
\hline 9 & 8 & 12.3 & 1.20 & 14.1 \\
\hline 9 & 10 & 13.3 & 0.42 & 23.1 \\
\hline 9 & 12 & 11.6 & 3.70 & 19.7 \\
\hline 9 & 14 & 6.7 & 4.63 & 27.1 \\
\hline 9 & 16 & 3.4 & 4.95 & 28.2 \\
\hline
\end{tabular}

Bond Type

Fusion Nugget

Fusion Nugget

Fusion Nugget

Fusion Nugget

Fusion Nugget

Recrystallized Interface

R. I. and Fusion Nugget

Fusion Nugget

Fusion Nugget

Fusion Nugget

Fusion Nugget

Fusion Nugget

Fusion Nugget

Fusion Nugget

Fusion Nugget

Fusion Nugget

R. I. and Fusion Nugget

R. I. and Fusion Nugget

R. I. and Fusion Nugget

R. I. and Fusion Nugget

Fusion Nugget

Table 6

0.020 Inch Diameter Alloy 180 Welded to 0.020 Inch Diameter Dumet.

\begin{tabular}{|c|c|c|c|c|c|}
\hline $\begin{array}{l}\text { Electrode } \\
\text { Force } \\
\text { (lbs.) }\end{array}$ & $\begin{array}{l}\text { Welding } \\
\text { Energy } \\
\text { (watt-sec.) }\end{array}$ & $\begin{array}{l}\text { Average } \\
\text { Strength } \\
\text { (lbs.) }\end{array}$ & $\begin{array}{l}\text { Standard } \\
\text { Deviation }\end{array}$ & $\begin{array}{c}\text { Average } \\
\text { Embedment } \\
\text { (percent wire diam.) }\end{array}$ & Bond Type \\
\hline 3 & 2 & 2.8 & 1.56 & 5.8 & Copper Braze \\
\hline 3 & 4 & 11.5 & 0.38 & 16.0 & Copper Braze \\
\hline 3 & 6 & 12.3 & 0.59 & 24.6 & Recrystallized Interface \\
\hline 3 & 8 & 10.6 & 1.89 & 32.8 & Fusion Nugget \\
\hline 5 & 2 & 1.1 & 0.81 & 8.2 & Copper Braze \\
\hline 5 & 4 & 11.3 & 0.48 & 14.7 & Copper Braze \\
\hline 5 & 6 & 12.2 & 0.66 & 25.0 & Recrystallized Interface \\
\hline 5 & 8 & 12.1 & 1.19 & 30.4 & Recrystallized Interface \\
\hline 7 & 4 & 12.0 & 0.62 & 17.0 & Recrystallized Interface \\
\hline 7 & 6 & 12.2 & 0.90 & 27.0 & Recrystallized Interface \\
\hline 7 & 8 & 11.4 & 0.89 & 35.2 & Recrystallized Interface \\
\hline 7 & 10 & 11.0 & 1.08 & 37.7 & Recrystallized Interface \\
\hline 7 & 12 & 9.6 & 1.03 & 39.2 & Recrystallized Interface \\
\hline 9 & 4 & 11.4 & 1.24 & 13.7 & Recrystallized Interface \\
\hline 9 & 6 & 11.7 & 0.79 & 27.0 & Recrystallized Interface \\
\hline 9 & 8 & 11.6 & 0.82 & 38.1 & Recrystallized Interface \\
\hline 9 & 10 & 11.0 & 1.12 & 34.4 & Recrystallized Interface \\
\hline 9 & 12 & 12.0 & 0.93 & 33.8 & Recrystallized Interface \\
\hline
\end{tabular}


Table 7

0.020 Inch Diameter Alloy 180 Welded to 0.020 Inch Diameter Kovar.

\begin{tabular}{|c|c|c|c|c|c|}
\hline $\begin{array}{l}\text { Electrode } \\
\text { Force } \\
\text { (Ibs.) }\end{array}$ & $\begin{array}{c}\text { Welding } \\
\text { Energy } \\
\text { (watt-sec.) }\end{array}$ & $\begin{array}{l}\text { Average } \\
\text { Strength } \\
\text { (lbs.) }\end{array}$ & $\begin{array}{l}\text { Standard } \\
\text { Deviation }\end{array}$ & $\begin{array}{c}\text { Average } \\
\text { Embedment } \\
\text { (percent wire diam.) }\end{array}$ & Bond Type \\
\hline 3 & 2 & 8.3 & 0.44 & 5.9 & Recrystallized Interface \\
\hline 3 & 4 & 11.5 & 0.53 & 10.9 & Recrystallized Interface \\
\hline 3 & 6 & 11.2 & 2.88 & 18.8 & Fusion Nugget \\
\hline 3 & 8 & 12.0 & 0.62 & 22.9 & Fusion Nugget \\
\hline 5 & 2 & 8.4 & 0.45 & 4.6 & Recrystallized Interface \\
\hline 5 & 4 & 11.5 & 0.47 & 15.1 & Fusion Nugget \\
\hline 7 & 2 & 8.7 & 0.53 & 7.0 & Recrystallized Interface \\
\hline 7 & 4 & 11.3 & 0.71 & 18.7 & Recrystallized Interface \\
\hline
\end{tabular}

Table 8

.020 Inch Diameter Alloy 180 Welded to .012 Inch by .022 Inch Alloy 180 Ribbon.

\begin{tabular}{|c|c|c|c|c|c|}
\hline $\begin{array}{c}\text { Electrode } \\
\text { Force } \\
\text { (lbs.) }\end{array}$ & $\begin{array}{c}\text { Welding } \\
\text { Energy } \\
\text { (watt-sec.) }\end{array}$ & $\begin{array}{c}\text { Average } \\
\text { Strength } \\
\text { (lbs.) }\end{array}$ & $\begin{array}{c}\text { Standard } \\
\text { Deviation }\end{array}$ & $\begin{array}{l}\text { Average } \\
\text { Micrometer } \\
\text { Reading } \\
\text { (mils) }\end{array}$ & $\begin{array}{c}\text { Embedment } \\
\text { (percent) }\end{array}$ \\
\hline 3 & 2 & 0.4 & $<.1$ & 31.3 & 2.2 \\
\hline 3 & 4 & 7.3 & .6 & 30.7 & 4.2 \\
\hline 3 & 6 & 9.8 & .8 & 29.5 & 7.8 \\
\hline 3 & 8 & 10.2 & 1.3 & 26.6 & 16.9 \\
\hline 3 & 10 & 9.6 & 1.0 & 23.7 & 26.0 \\
\hline 3 & 12 & 9.5 & 1.6 & 22.0 & 31.0 \\
\hline 5 & 4 & 4.7 & 1.8 & 30.0 & 6.3 \\
\hline 5 & 6 & 9.2 & 1.0 & 27.9 & 12.9 \\
\hline 5 & 8 & 10.6 & .9 & 26.0 & 18.6 \\
\hline 5 & 10 & 9.4 & 1.9 & 22.5 & 29.8 \\
\hline 5 & 12 & 10.4 & 1.2 & 21.4 & 33.2 \\
\hline 5 & 14 & 11.2 & .9 & 20.4 & 36.2 \\
\hline 5 & 16 & 11.1 & .8 & 20.6 & 35.8 \\
\hline 5 & 18 & 10.7 & 1.2 & 20.4 & 36.3 \\
\hline 7 & 4 & 2.8 & 1.3 & 29.2 & 8.9 \\
\hline 7 & 6 & 8.1 & .8 & 27.9 & 12.9 \\
\hline 7 & 8 & 10.7 & .8 & 25.9 & 19.0 \\
\hline 7 & 10 & 11.2 & 1.0 & 23.7 & 26.0 \\
\hline 7 & 12 & 10.9 & 1.4 & 22.2 & 30.8 \\
\hline 7 & 14 & 11.3 & .7 & 21.6 & 32.5 \\
\hline 7 & 16 & 11.4 & .5 & 20.2 & 37.0 \\
\hline 7 & 18 & 10.9 & 1.0 & 20.2 & 37.0 \\
\hline 9 & 6 & 6.5 & .5 & 27.9 & 12.7 \\
\hline 9 & 8 & 9.7 & .8 & 25.8 & 19.3 \\
\hline 9 & 10 & 10.9 & .6 & 23.5 & 26.4 \\
\hline 9 & 12 & 10.8 & 1.0 & 21.3 & 33.5 \\
\hline 9 & 14 & 11.0 & .5 & 21.5 & 32.7 \\
\hline 9 & 16 & 11.4 & .6 & 21.0 & 34.5 \\
\hline
\end{tabular}




\section{RESULTS AND DISCUSSION}

Tests to correlate embedment with tensile-shear strength and strength variation indicate that an optimum degree of embedment exists for each combination of materials investigated. The

Table 9

Optimum Embedment Values and Average Tensile-shear Strengths for Material Combinations Investigated.

\begin{tabular}{|l|c|c|}
\hline $\begin{array}{c}\text { Materials } \\
\text { (0.020 inch Diam.) }\end{array}$ & $\begin{array}{c}\text { Optimum } \\
\text { Embedment } \\
\text { (percent) }\end{array}$ & $\begin{array}{c}\text { Average } \\
\text { Tensile-shear } \\
\text { Strength } \\
\text { (pounds) }\end{array}$ \\
\hline Nickel 200-Nickel 200 & 13.0 & 16.4 \\
Nickel 200-Dumet & 17.5 & 14.5 \\
Nickel 200-Kovar & 14.0 & 11.8 \\
Nickel 200-Alloy 180 & 15.0 & 9.9 \\
Alloy 180-Alloy 180 & 22.0 & 12.1 \\
Alloy 180-Dumet & 18.0 & 11.8 \\
Alloy 180-Kovar & 10.0 & 10.6 \\
Alloy 180- & & \\
Alloy 180 Ribbon & $18.0 *$ & 10.7 \\
\hline
\end{tabular}

*Measured with micrometers.

\section{Nickel 200 Welded to Nickel 200}

Nickel 200 was welded to itself over a wide range of welder variables. Throughout the useful range of welder variables, it was found that the weld strength increases with increasing embedment (Figure 7) and pull strengths up to 20 pounds can be achieved before excessive spitting or electrode sticking occurs. As shown in Figure 8 , the minimum standard deviation occurs at approximately 13 percent embedment. The average pull strength at this embedment is 16.4 pounds, somewhat lower than the maximum attainable. Embedment can be varied by optimum degree of embedment was found to be between 10 and 25 percent of the wire diameter for all material combinations tested (Table 9).

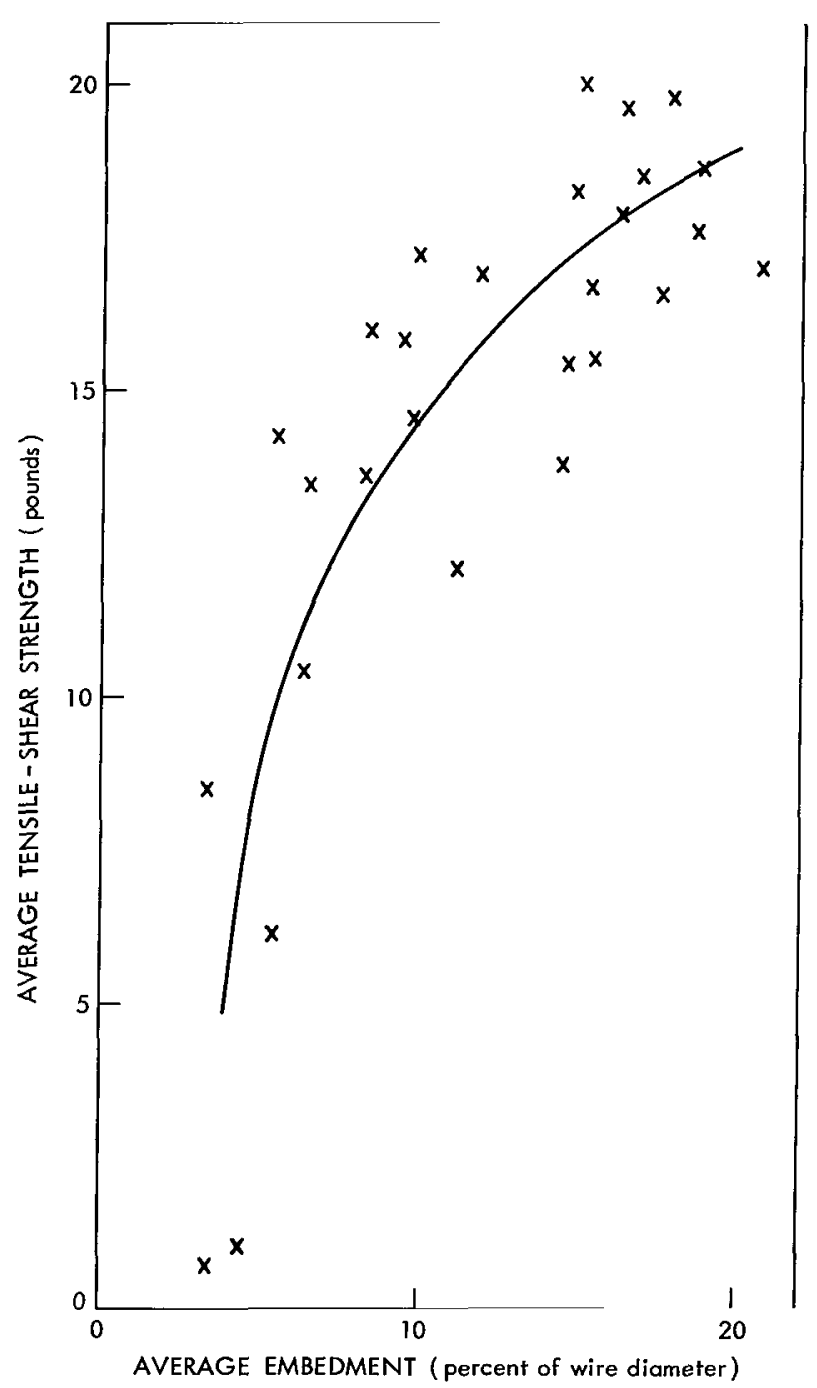

Figure 7-Average embedment plotted as a function of average tensile-shear strength for 0.020 inch diameter Nickel 200 welded to 0.020 inch diameter Nickel 200 . 


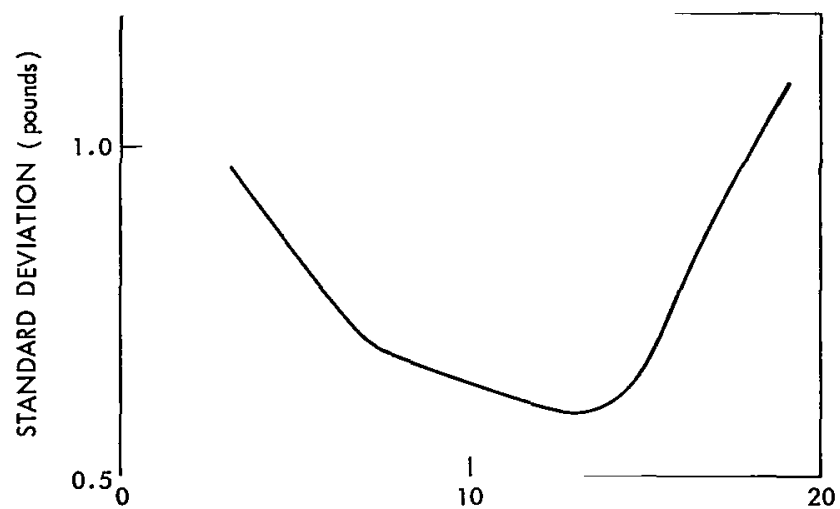

AVERAGE EMBEDMENT (percent of wire diameter)

Figure 8-Average embedment plotted as a function of standard deviation for 0.020 inch diameter Nickel 200 welded to 0.020 inch diameter Nickel 200 .

changing either the electrode force or the welding energy. An increase in weld energy produces greater embedment; at low electrode force settings, an increase in the electrode force produces decreased embedment. At any useful value of weld energy, as shown in Figure 9, a 3-pound electrode force will produce a greater embedment than a 9-pound force. This is attributed to the difference in the resistance of the joints, and hence, the heat developed in the joints. A high electrode force deforms the wire surfaces at their points of contact, providing more surface area to transmit the electrical energy. The lower resistance

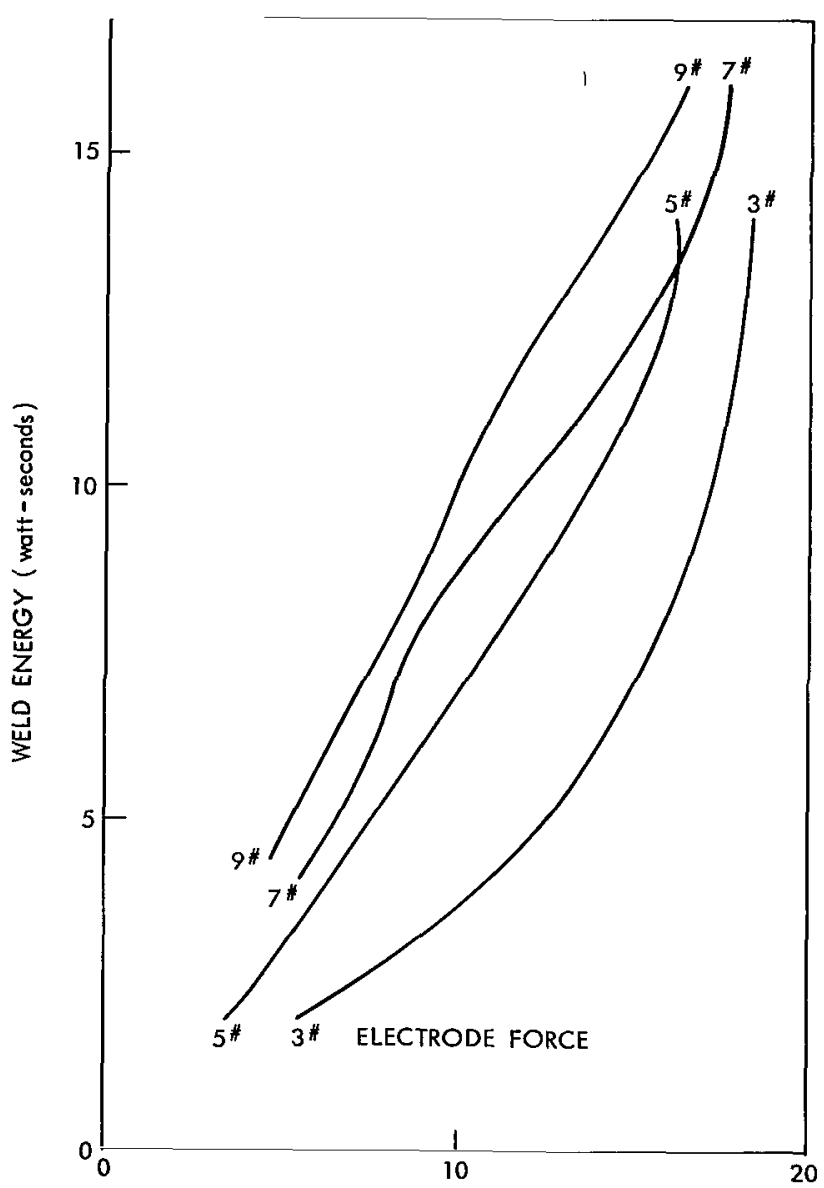

AVERAGE EMBEDMENT (percent of wire diameter)

Figure 9-Average embedment plotted as a function of welding energy for 0.020 inch diameter Nickel 200 welded to 0.020 inch diameter Nickel 200. in the joint produces less heat, according to the relationship $H \propto I^{2} R t$, where $H$ is the energy (or heat) in watt-seconds, $I$ is the current in amperes, $R$ is the resistance in ohms, and $t$ is the time in seconds. A proportionality constant $\mathrm{K}$ can be inserted to convert this relationship to the equation $H=I^{2}$ RtK. The constant $K$ is dimensionless, usually less than unity, and is dependent upon the electrical and thermal properties of the materials to be welded. The resistance $\mathbf{R}$ is determined principally at the junction of the two materials to be welded. $A$ high electrode force will reduce the interfacial resistance $\mathrm{R}$ and consequently will lower the energy $\mathrm{H}$ dissipated in the joint. Hence, at the same welding energy level, higher electrode forces cause lower interfacial resistance and less heat to be produced in the joint, resulting in a decreased embedment. This relationship is mentioned because under particular conditions, as shown later, a seemingly opposite effect can be encountered. A photomicrograph of a typical joint is shown in Figure 10. 


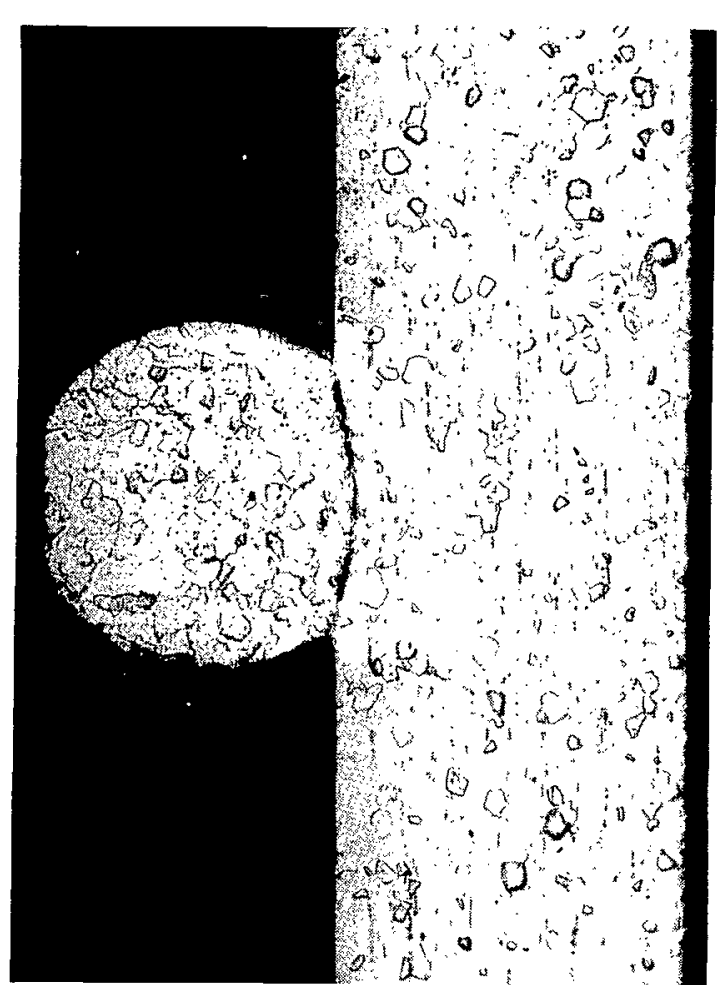

Figure 10-Photomicrograph of a typical crosswire weld joint in Nickel 200 (magnified 70X).

\section{Nickel 200 Welded to Dumet}

Figure 11 shows that the maximum strength in joints of Nickel 200 to Dumet is obtained at an embedment of 19 percent of the wire diameter. Welds with greater embedment have lower strength and less reliability. Figure 12 indicates that Nickel 200 to Dumet joints are less consistent than those of Nickel 200 to Nickel 200. The optimum standard deviation occurs at approximately $\mathbf{1 7 . 5}$ percent embedment. Although this embedment does not coincide with the minimum standard deviation value, it is an embedment value representing good strength (14.5 pounds) and better consistency than welds exhibiting slightly more or less embedment. The low standard deviation values below 12 percent embedment represent copper braze type joints of less than 10 pounds average pull strength. The initial increase in the standard deviation values for up to 12 percent embedment

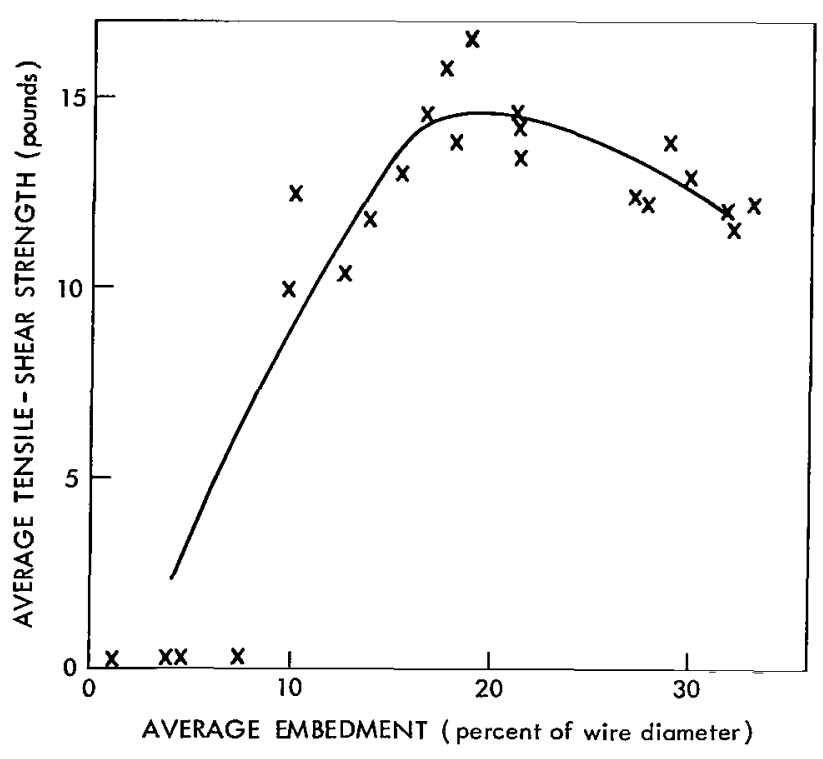

Figure 11-Average embedment plotted as a function of average tensile-shear strength for 0.020 inch diameter Nickel 200 welded to 0.020 inch diameter Dumet.

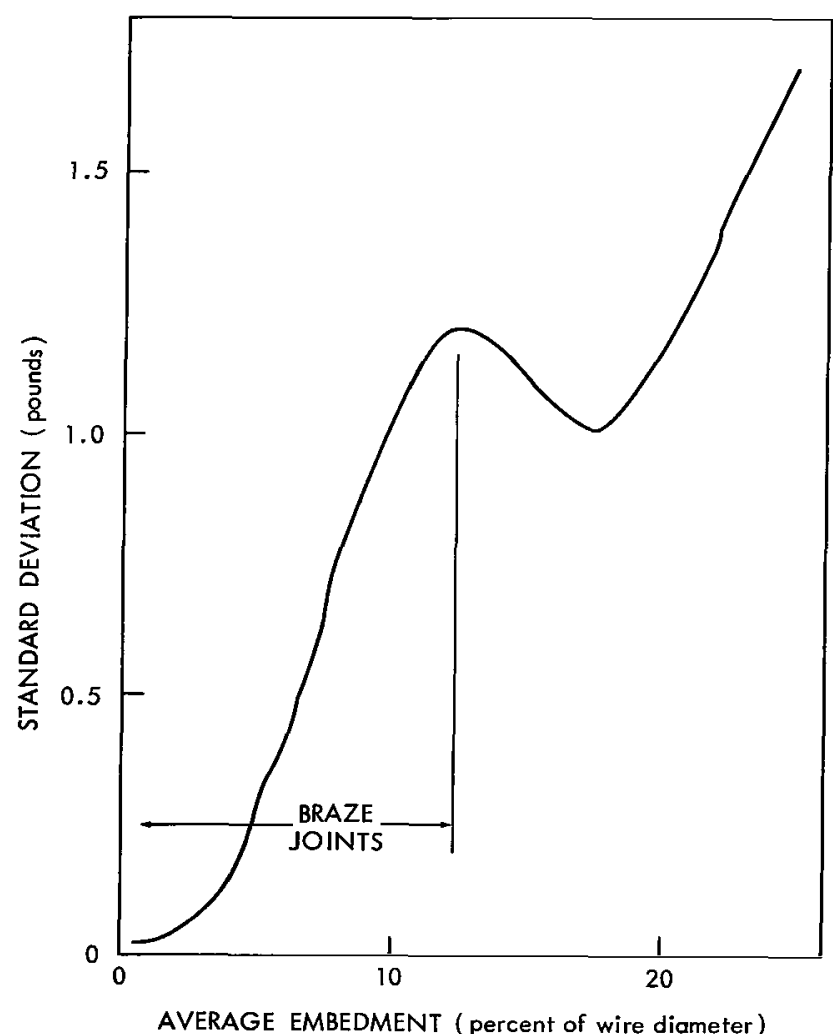

Figure 12-Average embedment plotted as a function of standard deviation for 0.020 inch diameter Nickel 200 welded to 0.020 inch diameter Dumet. 
occurs from braze type joints having increasing average strengths but decreasing consistencies. Above 12 percent embedment all welds exhibit a recrystallized interface or forge type bond as in Figure 13. At embedments greater than the bond type transition value, the standard deviation versus embedment curve follows the same pattern as that of Nickel to Nickel welds (Figure 7), a "U" shaped curve exhibiting only one minimum. The influence of welding energy upon the embedment is shown in the constant electrode force curves of Figure 14. At low welding energies, the joints produced at high electrode forces displayed low embedments while lower electrode forces caused higher embedments. At higher welding energies, however, the higher electrode forces caused greater embedment than was exhibited in welds made at the lower electrode forces. The crossing of the constant force curves seems to be related to the inability of the increasing electrode forces to provide lower interfacial resistance beyond the point where there are negligible increases in the flattening of interfacial surfaces. At forces above this value, the heat produced in the joint becomes independent of the electrode force and is controlled only by the welder's energy setting. The embedment, however, is not independent of the electrode force. The welding energy is applied to the joint over a period of several milliseconds, as shown by oscilloscope traces. During the time when the energy is sufficient to cause melting or extreme softening, the movable electrode is accelerating at a rate determined by its inertia, electrode force, and resisting force. At high electrode forces, the electrode acceleration is large enough to cause some embedment while the energy is being applied. This effectively drives the softened or melted zone into the materials, thus permitting greater embedments to be produced as the electrode force is increased.

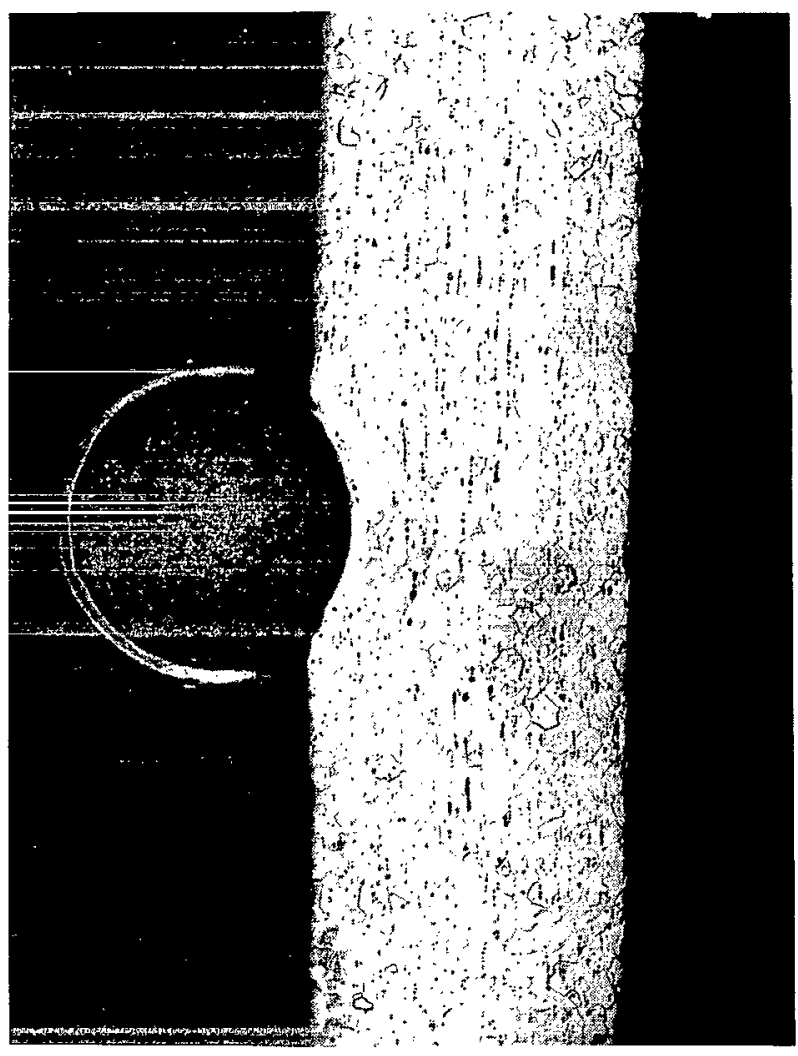

Figure 13-Photomicrograph of a cross-wire weld joint between Dumet (left) and Nickel 200 magnified 70X).

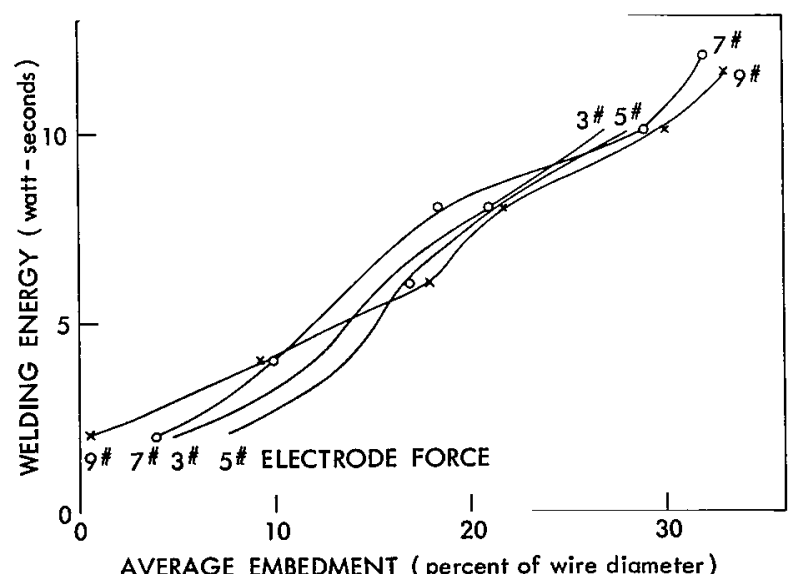

Figure 14-Average embedment plotted as a function of welding energy for 0.020 inch diameter Nickel 200 welded to 0.020 inch diameter Dumet. 


\section{Nickel 200 Welded to Kovar}

Figure 15 illustrates the strengthembedment relationship for Nickel welded to gold plated Kovar. As with other materials, an embedment exists where the strength is near maximum and where the standard deviation is minimum. Figure 16 shows that the minimum standard deviation occurs at an embedment of 14 percent. The average tensileshear strength at this embedment is 11.8 pounds which is near the maximum strength of $\mathbf{1 2 . 5}$ pounds. Embedment is plotted as a function of welding energy in Figure 17 for the values of electrode forces used. At low energies, the embedment is somewhat independent of the electrode force. This is a result of the material's hardness which allows little deformation of the contact surfaces before the energy is applied. As the welding energy is increased, the electrode force becomes more effective in producing an embedment because the molten or softened zone is carried forward by the moving wire. A photomicrograph of a typical joint is shown in Figure 18.

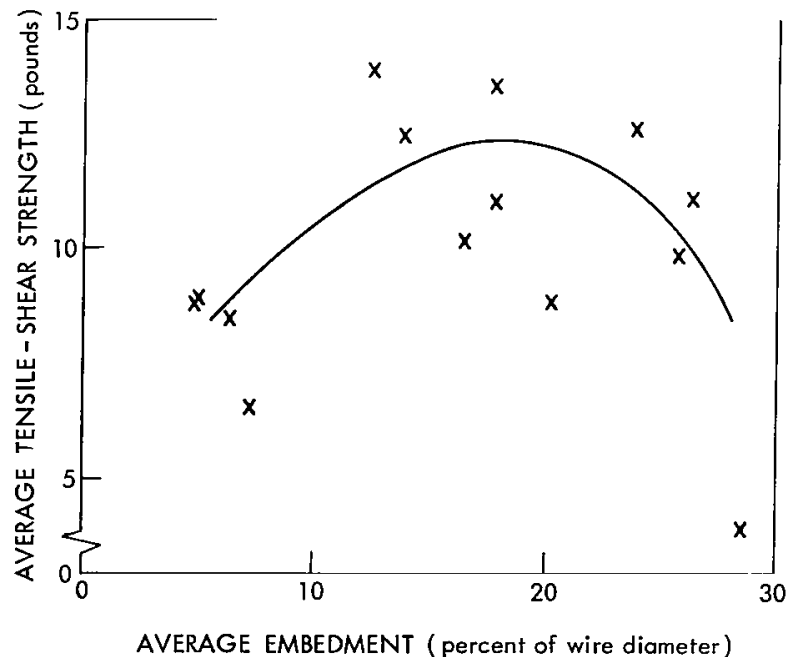

Figure 15-Average embedment plotted as a function of average tensile-shear strength for 0.020 inch diameter Nickel 200 welded to 0.020 inch diameter Kovar.

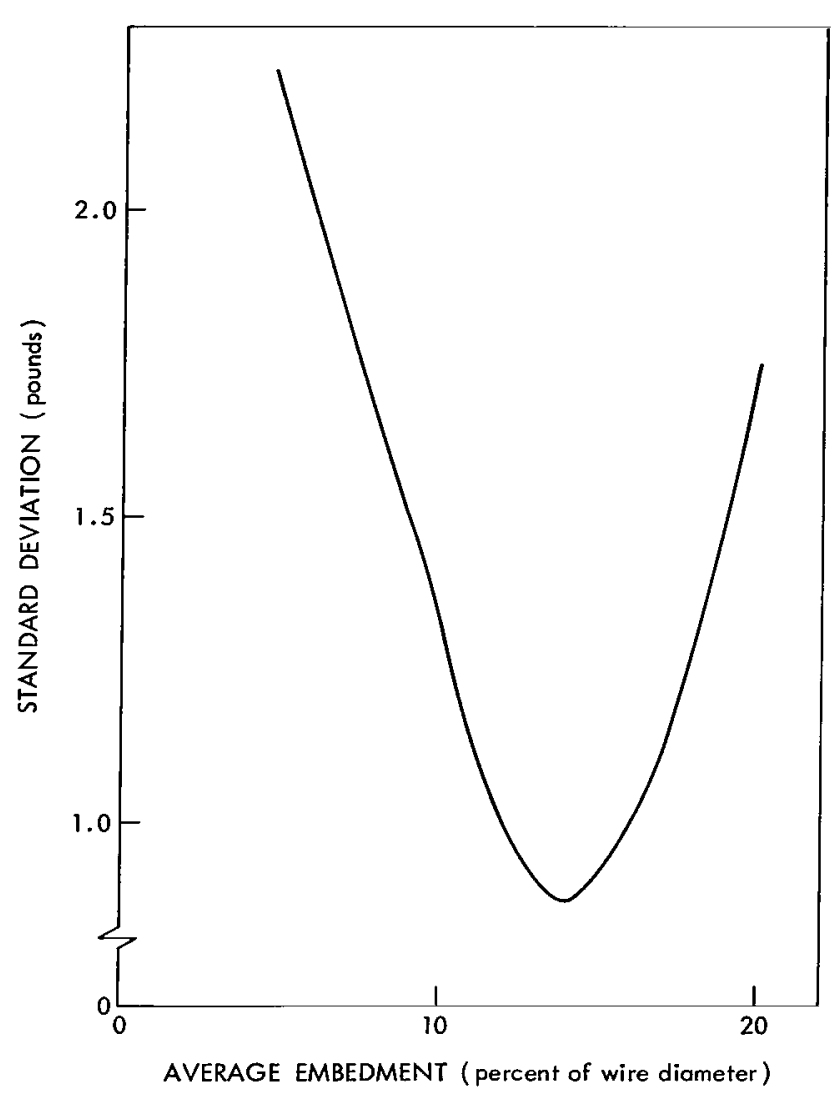

Figure 16-Average embedment plotted as a function of standard deviation for 0.020 inch diameter Nickel 200 welded to 0.020 inch diameter Kovar.

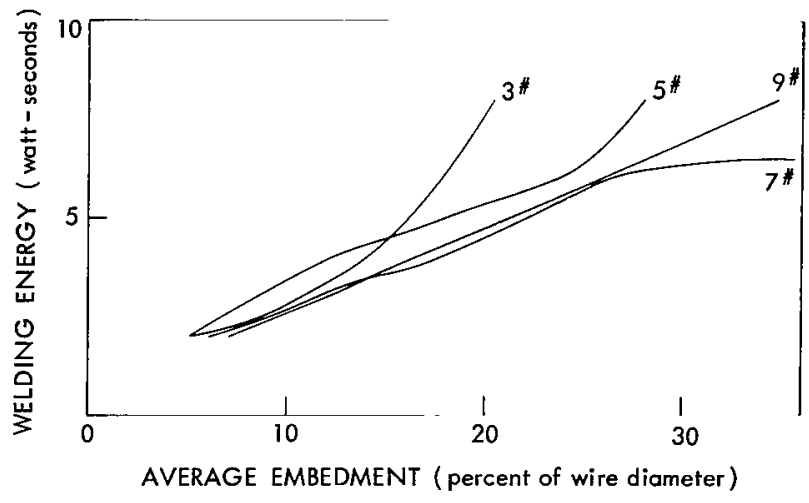

Figure 17-Average embedment plotted as a function of welding energy for 0.020 inch diameter Nickel 200 welded to 0.020 inch diameter Kovar. 

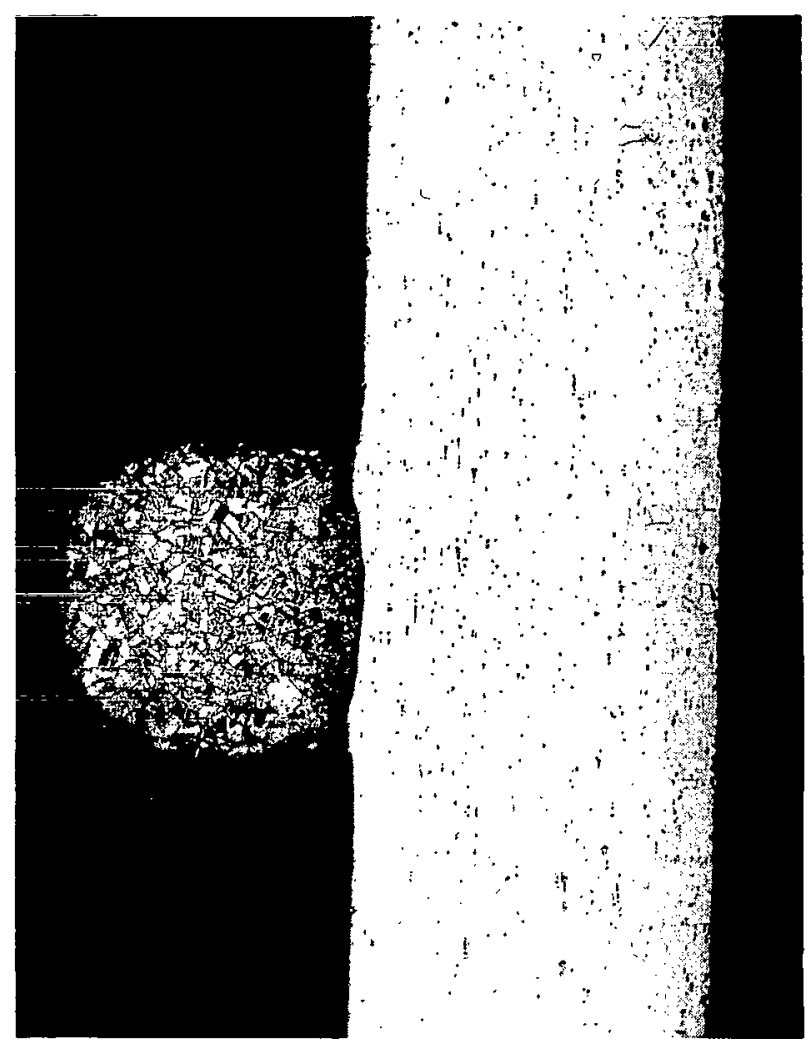

Figure 18-Photomicrograph of a cross-wire weld joint between nickel (right) and gold plated Kovar (magnified 70X).

\section{Nickel 200 Welded to Alloy 180}

Nickel welded to Alloy 180 exhibits the same characteristic strength-embedment curve as described previously. The maximum average strength of 10.0 pounds occurs at an embedment of 16 percent (Figure 19) and the standard deviation reaches a minimum at 15 percent embedment (Figure 20). The optimum weld schedule would then produce joints having embedments near 15 percent. Figure 21 illustrates the small dependence of embedment upon electrode force. In the energy range required to produce approximately 15 percent embedment, the joint strength (and embedment) is dependent only upon the welding energy.

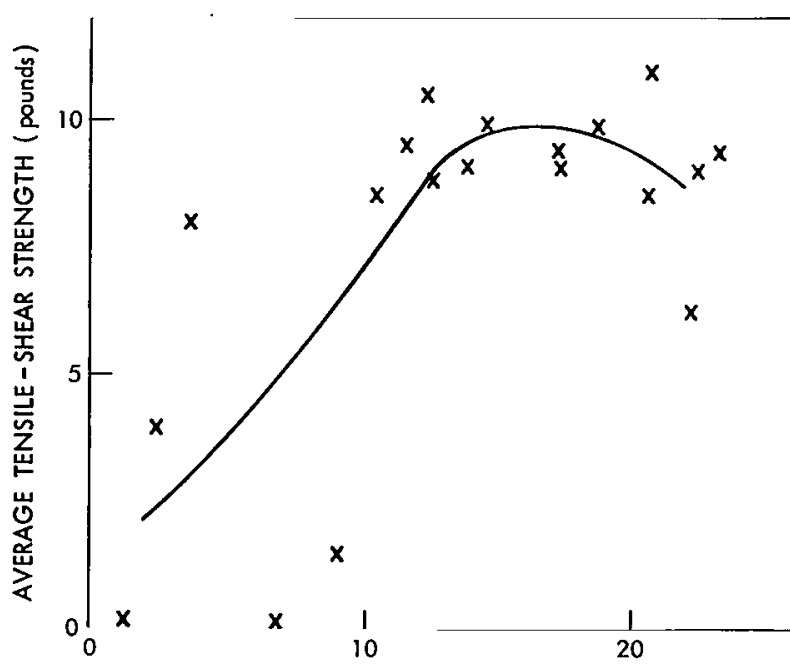

AVERAGE EMBEDMENT (percent of wire diameter)

Figure 19-Average embedment plotted as a function of average tensile-shear strength for 0.020 inch diameter Nickel 200 welded to 0.020 inch daimeter Alloy 180.

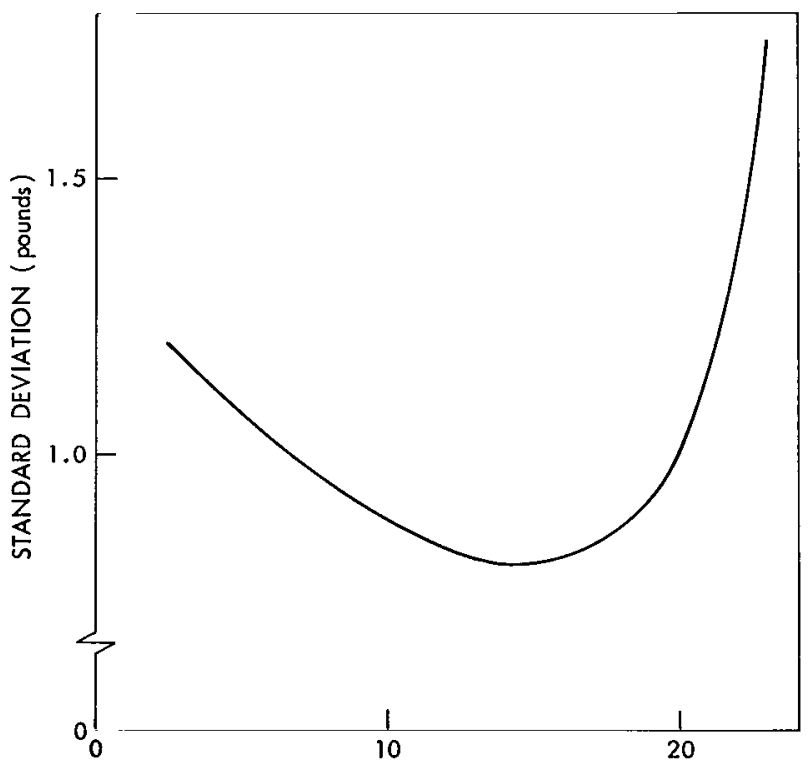

AVERAGE EMBEDMENT (percent of wire diameter)

Figure 20-Average embedment plotted as a function of standard deviation for 0.020 inch diameter Nickel 200 welded to 0.020 inch diameter Alloy 180. 
Alloy 180 exhibits a considerably lower hardness value than Nickel 200, Kovar, or Dumet. This allows a greater deformation to be obtained from the initial electrode force. Due to its lower melting point and thermal conductivity, fusion nugget bonds readily occur in joints consisting of Alloy 180 and other common lead materials. A photomicrograph of a typical joint is shown in Figure 22.

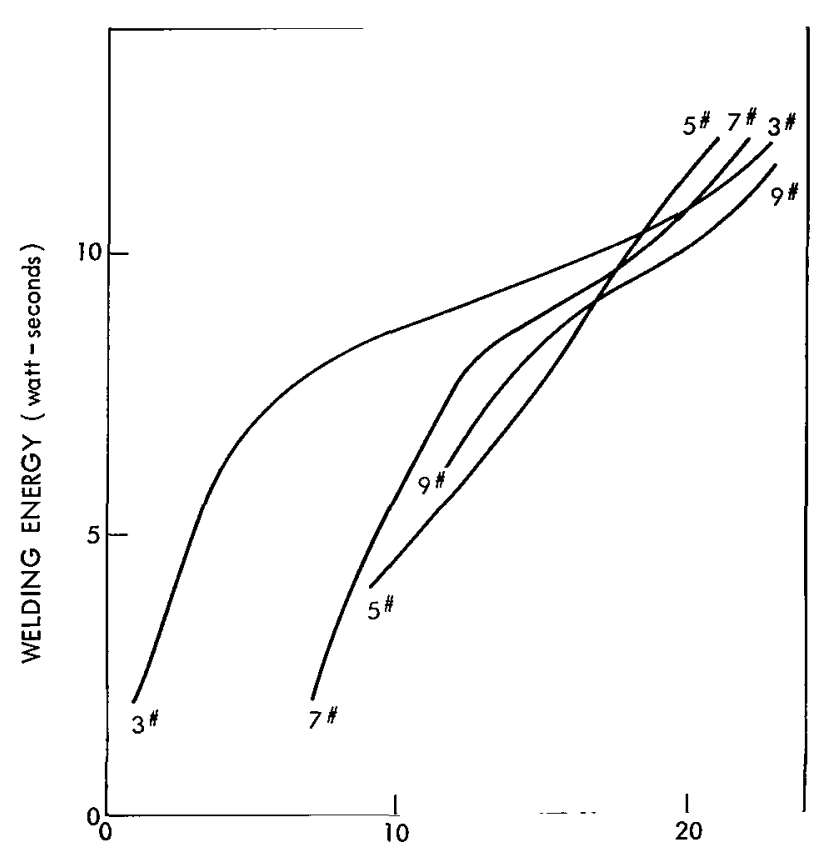

AVERAGE EMBEDMENT (percent of wire diameter)

Figure 21 -Average embedment plotted as a function of of welding energy for 0.020 inch diameter Nickel 200 welded to 0.020 inch diameter Alloy 180.

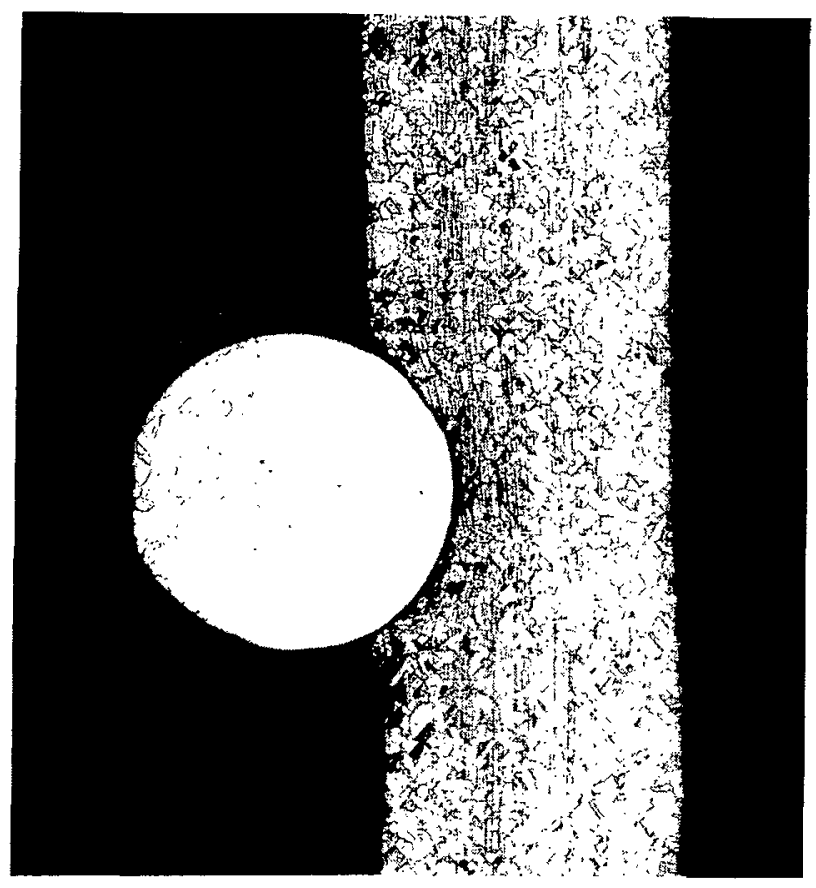

Figure 22-Photomicrograph of a cross-wire weld joint between nickel (left) and Alloy 180. The majority of the total embedment occurs in the softer Alloy 180 (magnified 70X).

\section{Alloy 180 Welded to Alloy 180}

Alloy 180 welded to itself generally produces sound welds with good reproducibility over a wide range of welder variables. A typical weld joint is shown in Figure 23. Figure 24 indicates a normal strength-embedment relationship with a maximum average strength of 12.1 pounds occurring at 22 percent embedment. Figure 25 shows that this embedment also corresponds to the best reproducibility. Slightly higher embedments produce a catastrophic increase in the standard deviation and a corresponding decrease in the average tensile-shear strength.

The most favorable welder settings produce welds having embedments slightly less than 22 percent and strengths slightly lower than the maximum in order to avoid welds having erratic strength values due to normal changes in the operational variables. Figure 26 illustrates the result of a change in an operational variable. The samples which produce the energy-embedment 


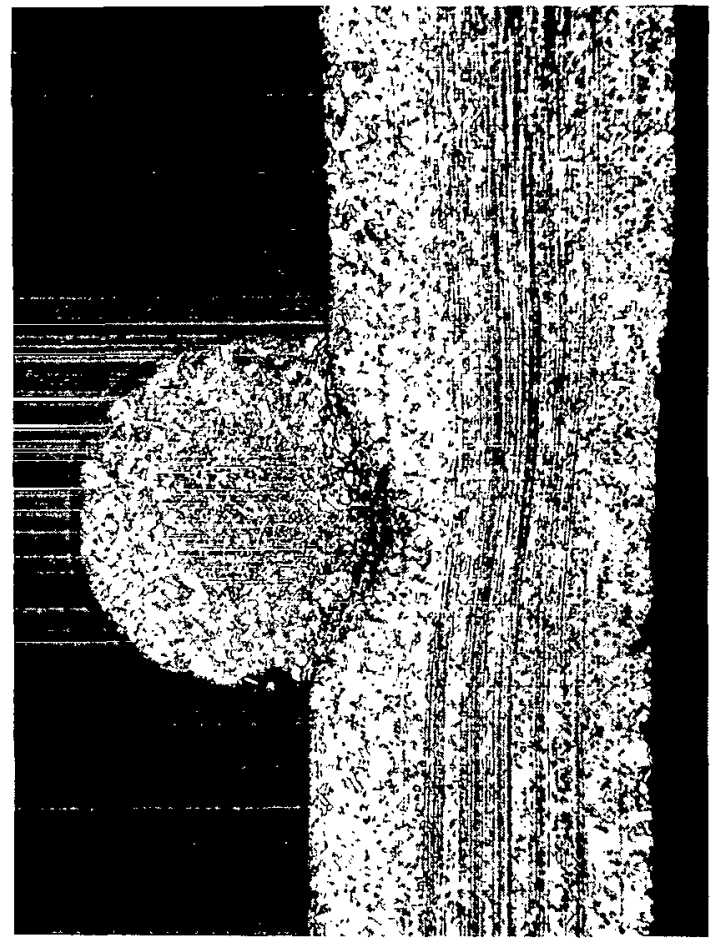

Figure 23-Photomi crograph of a typical crosswire weld joint of Alloy 180 (magnified 70X).

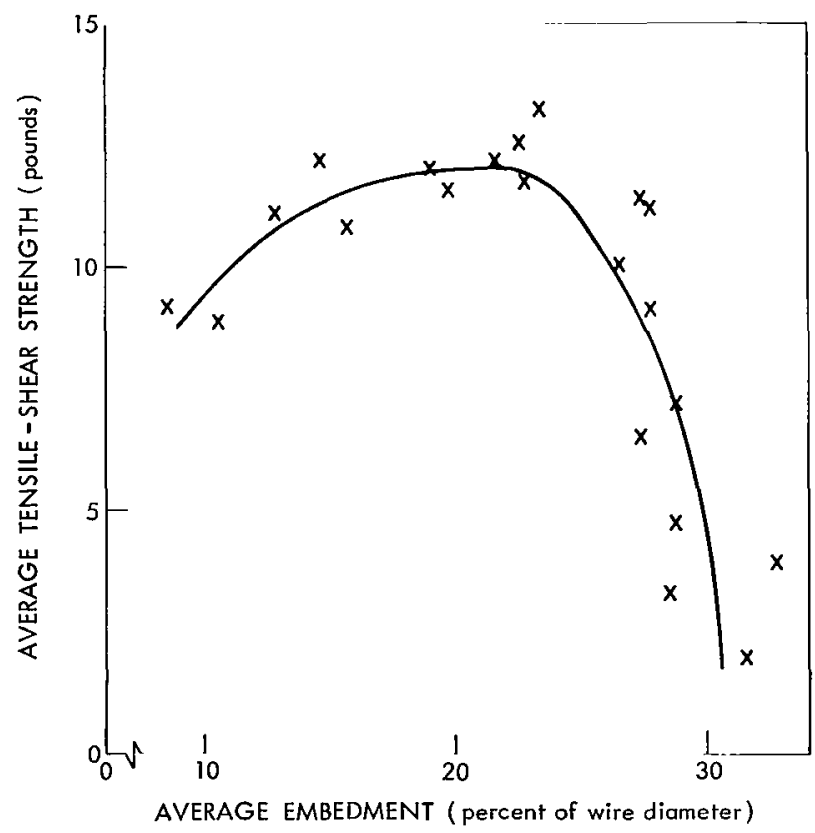

Figure 24-Average embedment plotted as a function of average tensile-shear strength for 0.020 inch diameter Alloy 180 welded to 0.020 inch diameter Alloy 180 .

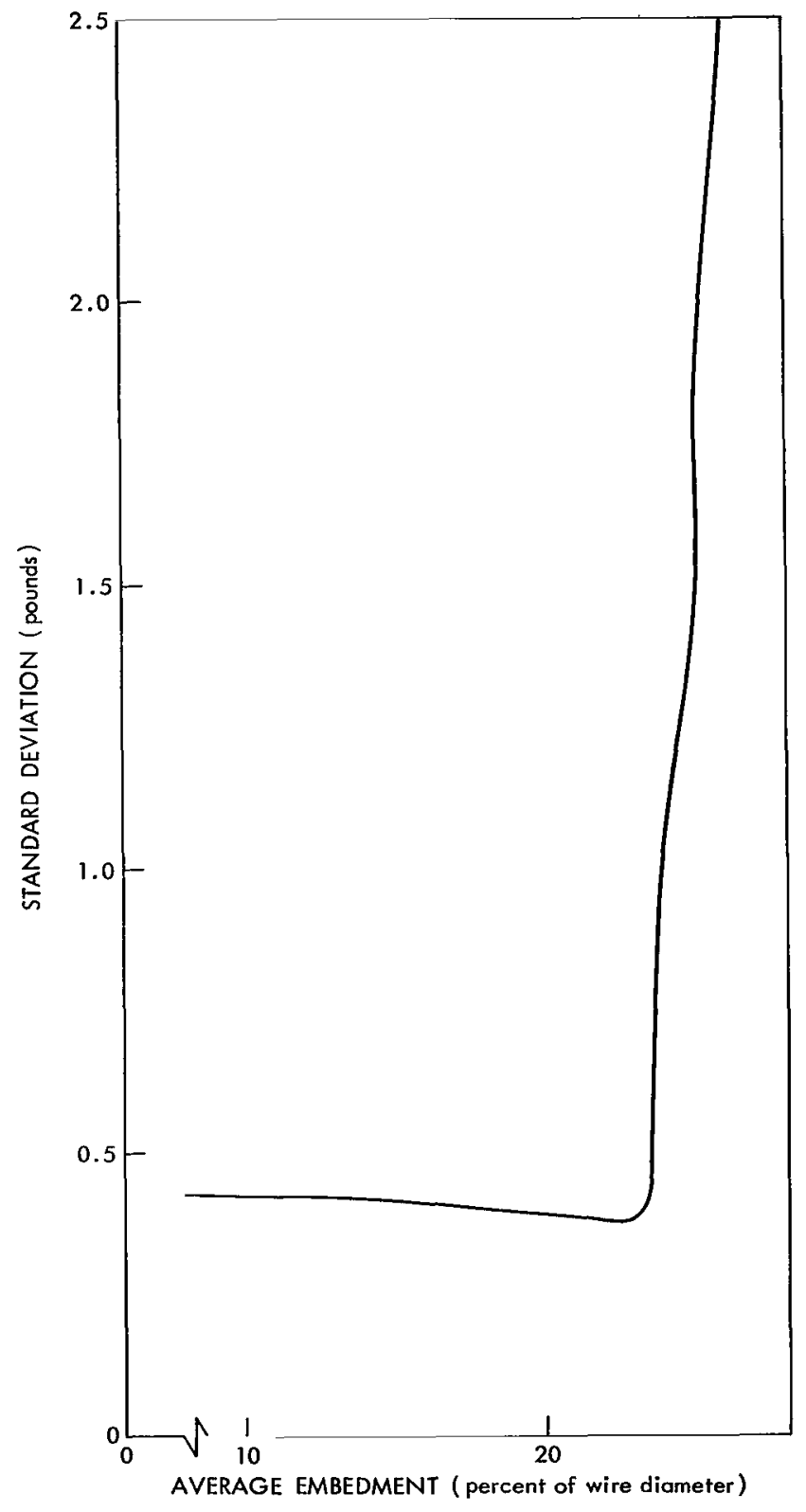

Figure 25-Average embedment plotted as a function of standard deviation for 0.020 inch diameter Alloy 180 welded to 0.020 inch diameter Alloy 180 . 
data were made on the same day and by the same operator, but the samples having high embedment were made on the following day. A change in some welder variable caused a different energy-embedment relationship. At the most practical embedment value (near 20 percent) more energy is required at the high electrode force settings in order to produce a given embedment. This again can be attributed to the lower interfacial resistance values at high electrode force settings.

\section{Alloy 180 Welded to Dumet}

At low welding energies and low electrode forces, Alloy 180 produces braze type joints with the copper sheath on the Dumet. These joints are low in strength and have poor reproducibility, therefore, the welding energy should be increased until braze joints are no longer obtained. Figure 27 illustrates the strength level obtainable in brazed joints. A maximum of 12.2 pounds average tensileshear strength was obtained, but with a slight increase in standard deviation. Figure 28 shows a minimum standard deviation at 18 percent embedment with an average tensileshear strength of 11.8 pounds.

Again, the samples exhibiting high embedments were made on a different day. Some change in conditions had caused a higher energy setting to be required in order to produce a given embedment (Figure 29). The average tensile-shear strength and standard deviation curves remain unaffected because the energy setting required to produce a particular embedment was not a consideration. A typical weld joint is shown in Figure 30.

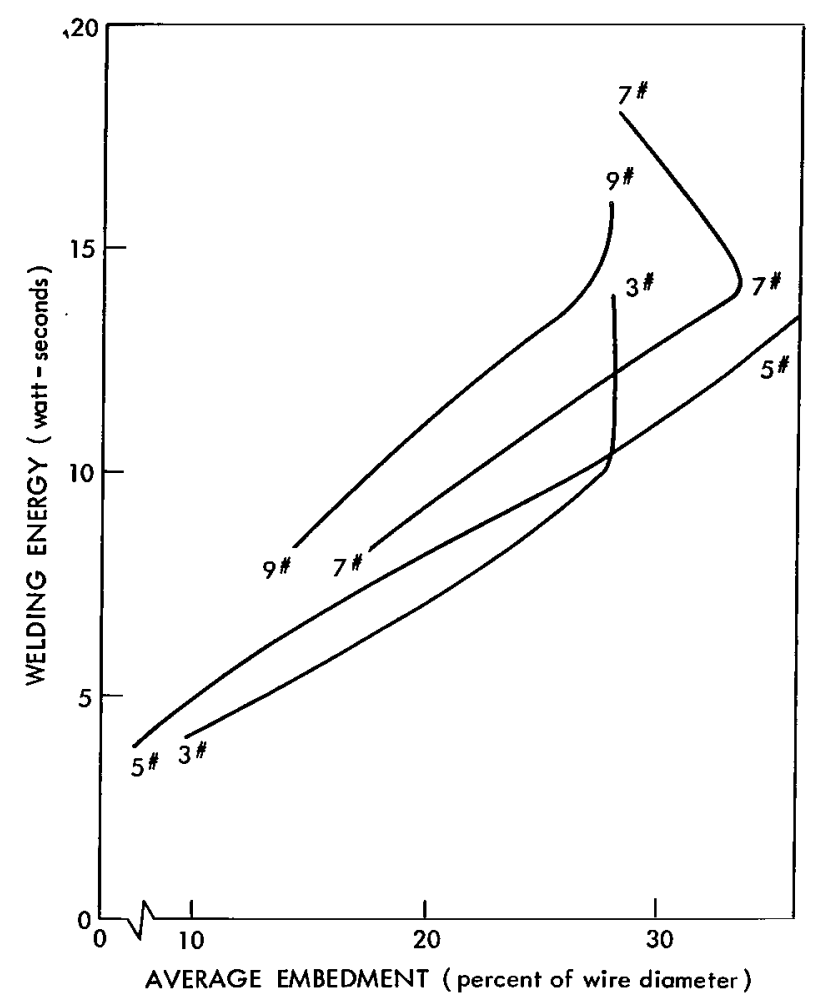

Figure 26-Average embedment plotted as a function of welding energy for 0.020 inch diameter Alloy 180 welded to 0.020 inch diameter Alloy 180.

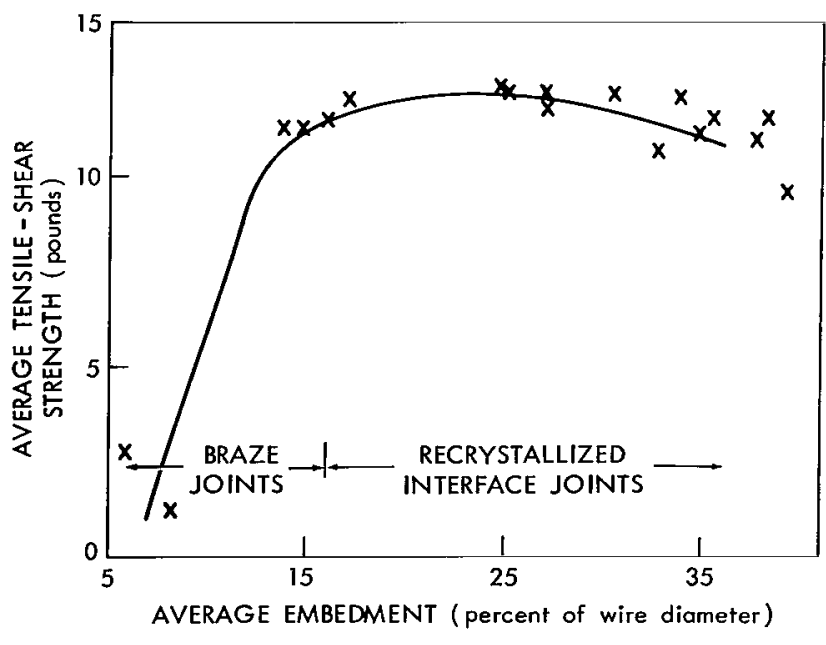

Figure 27-Average embedment plotted as a function of average tensile-shear strength for 0.020 inch diameter Alloy 180 welded to 0.020 inch diameter Dumet. 


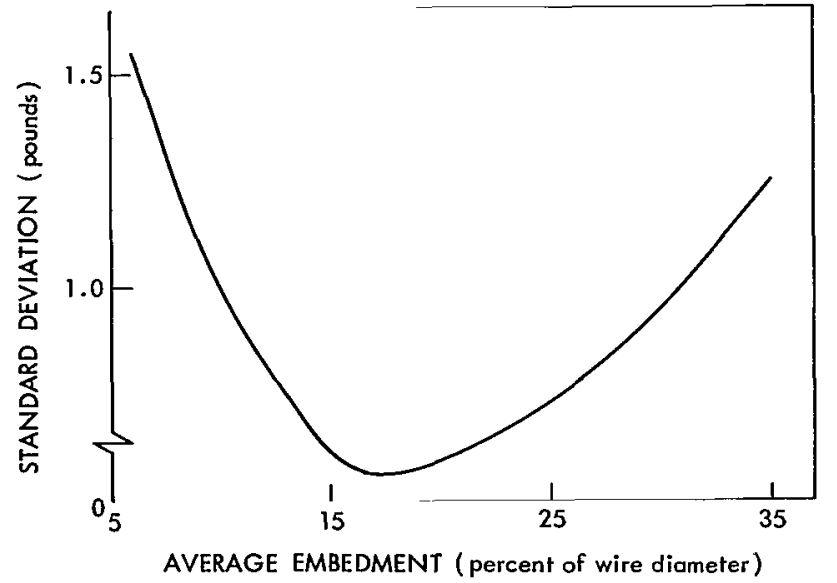

Figure 28-Average embedment plotted as a function of standard deviation for 0.020 inch diameter Alloy 180 welded to 0.020 inch diameter Dumet.

\section{Alloy 180 Welded to Kovar}

Kovar, when welded to Alloy 180, produces sound welds over a narrow range of electrode forces and welding energies. No welds were obtained when embedments were less than 4.6 percent, and excessive sticking and spitting occurred at settings which produced more than 23 percent embedment (Figure 31 ). The standard deviation rises sharply as the embedment is increased (Figure 32), however, the average strength level remains essentially constant above 10 percent embedment. Because of the limited range of welder settings which produce sound welds, there was not enough data to produce a conclusive set of welding energy versus embedment curves for this alloy combination. Figure 33 presents the limited data that was produced and Figure 34 shows a typical weld joint.

\section{Alloy 180 Welded to Alloy 180 Ribbon}

Micrometer measurements of forty-eight embedments at each machine setting indicate that the strength can be correlated with the joint

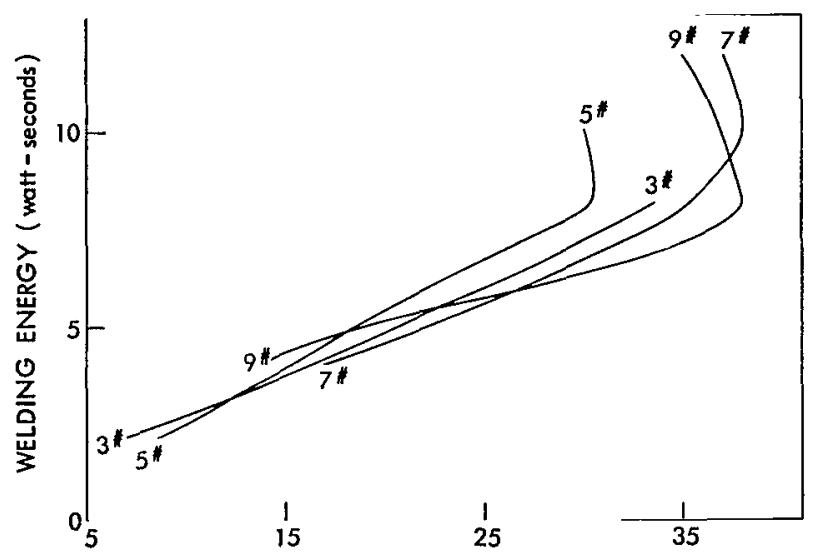

AVERAGE EMBEDMENT (percent of wire diameter)

Figure 29-Average embedment plotted as a function of welding energy for 0.020 inch diameter Alloy 180 welded to 0.020 inch diameter Dumet.

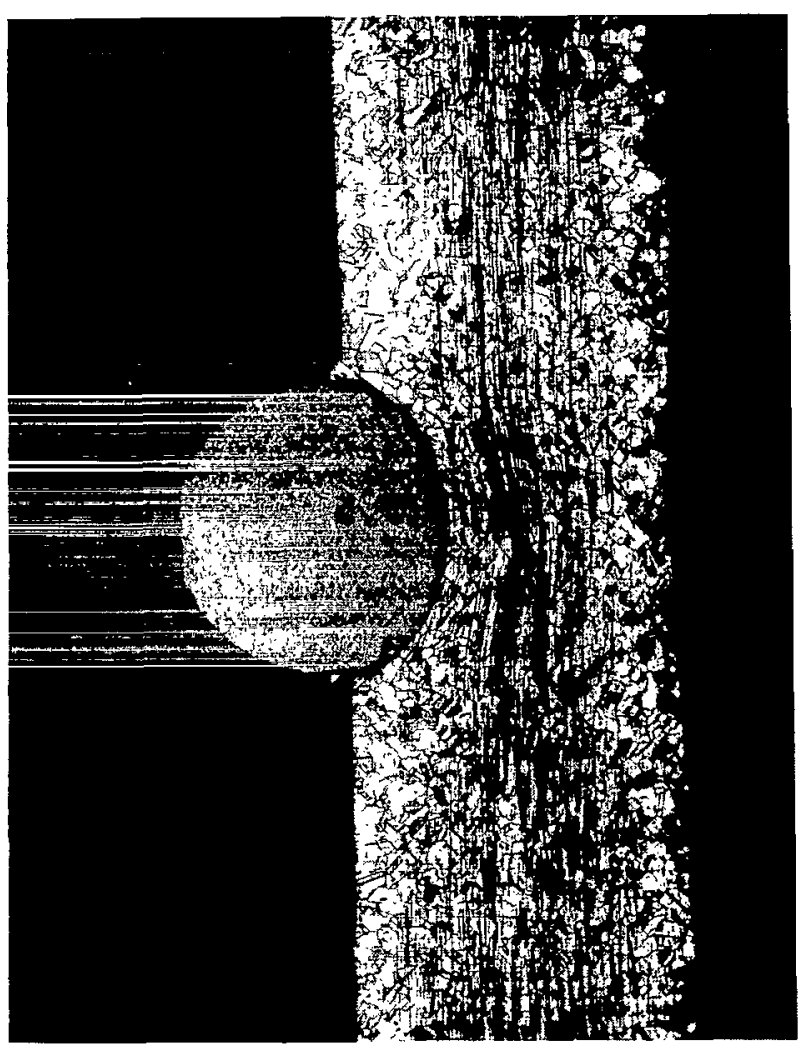

Figure 30-Photomicrograph of a cross-wire weld joint between Dumet (left) and Alloy 180 (magnified 70X). 


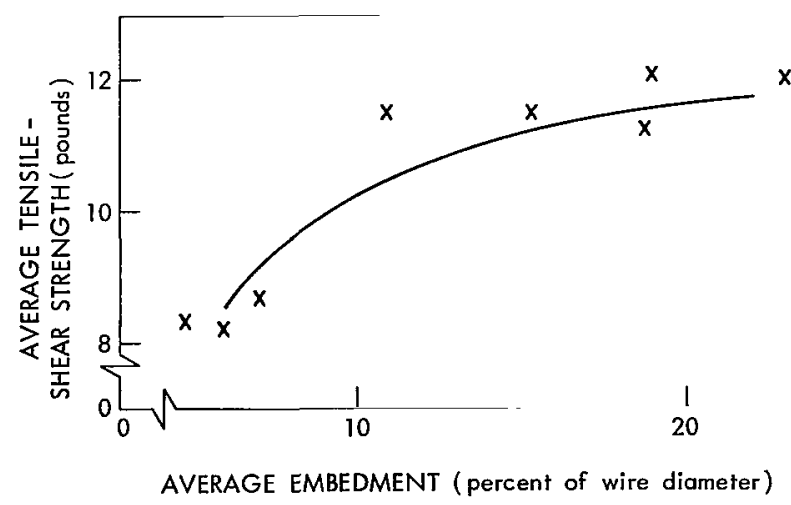

Figure 31-Average embedment plotted as a function of average tensile-shear strength for 0.020 inch diameter Alloy 180 welded to 0.020 inch diameter Kovar.

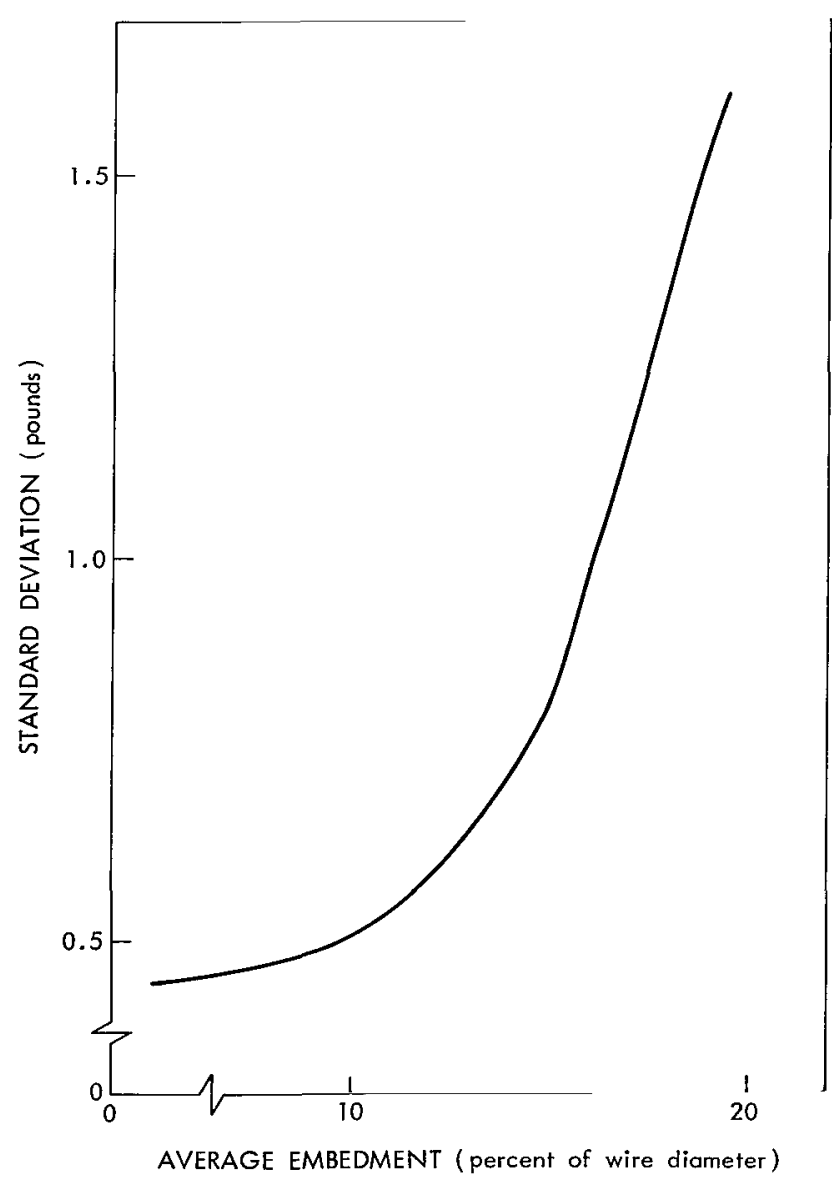

Figure 32-Average embedment plotted as a function of standard deviation for 0.020 inch diameter Alloy 180 welded to 0.020 inch diameter Kovar.

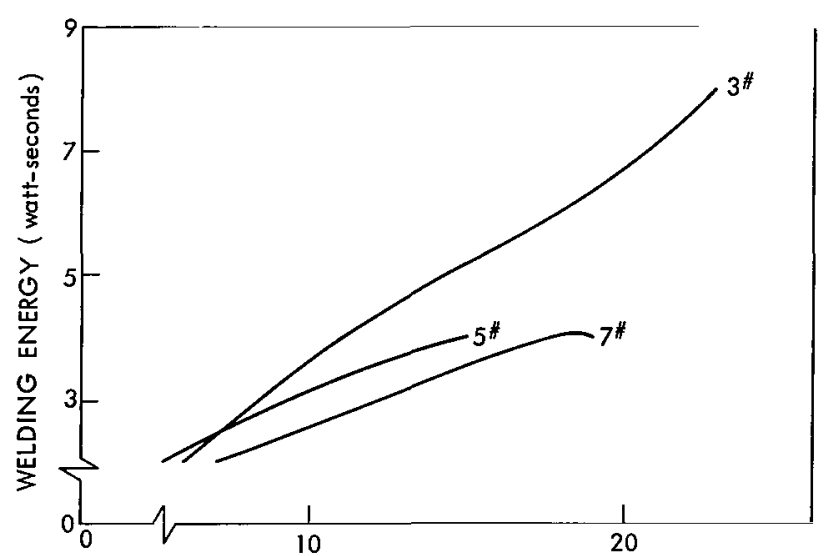

AVERAGE EMBEDMENT (percent of wire diameter)

Figure 33-Average embedment plotted as a function of welding energy for 0.020 inch diameter Alloy 180 welded to 0.020 inch diameter.

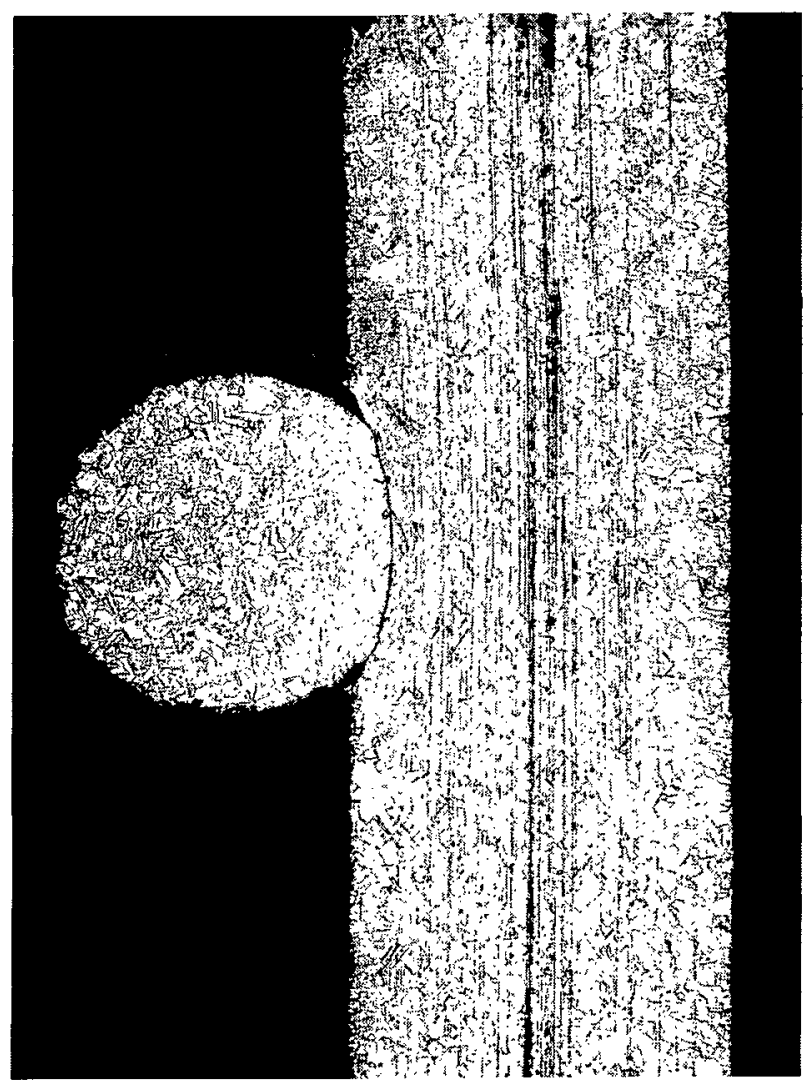

Figure 34-Photomicrograph of a cross-wire weld joint between gold plated Kovar (left) and Alloy 180 (magnified $70 X$ ). 
thickness. Even when welding ribbon to wire, which is a case where embedment cannot be assumed to occur equally in each member, the total joint thickness measurement is of value as a nondestructive method of monitoring joint quality. It is important, however, to maintain normal quality control practices. Embedment measurements can be employed as an additional check in a production module if work space permits. In addition, the strength-standard deviation-embedment relationships must be determined previously in order for the embedment data to be useful.

Figure 35 presents the strength-embedment relationship for Alloy 180 wire $(0.020$ inch diameter) welded to Alloy 180 ribbon (0.012 inch by 0.022 inch). The joint strength tends to increase with embedment up to and above 35 percent. However, the high weld energy settings which are required to produce embedments over 25 percent create spitting, sticking of the joint members to the electrodes, and excessive surface identation. The embedment produced is highly dependent upon the weld energy, but virtually independent of the electrode force. Since the ribbon member presents a surface 0.022 inch in width to the wire member, extremely high electrode forces are required to produce a change in the interfacial contact area. The trend of embedments with welding energies at constant electrode forces is illustrated in Figure 36.

\section{CONCLUSIONS}

1. Optimum strength and standard deviation values are obtained at a particular degree of embedment, depending upon the alloy combination and the wire sizes.

2. An increase in weld energy is more effective than a change in electrode force in order to produce an increased embedment.

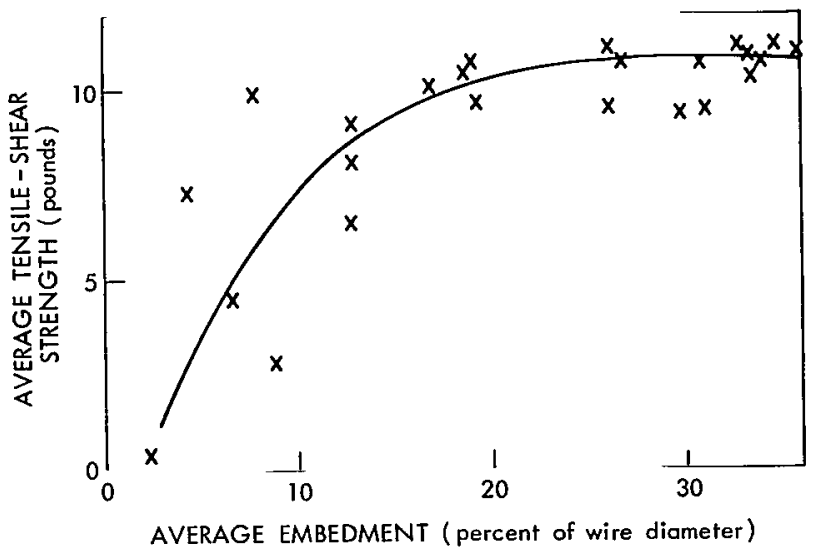

Figure 35-Average embedment plotted as a function of average tensile-shear strength for 0.020 inch diameter Alloy 180 welded to 0.012 inch $\times 0.022$ inch Alloy 180 ribbon.

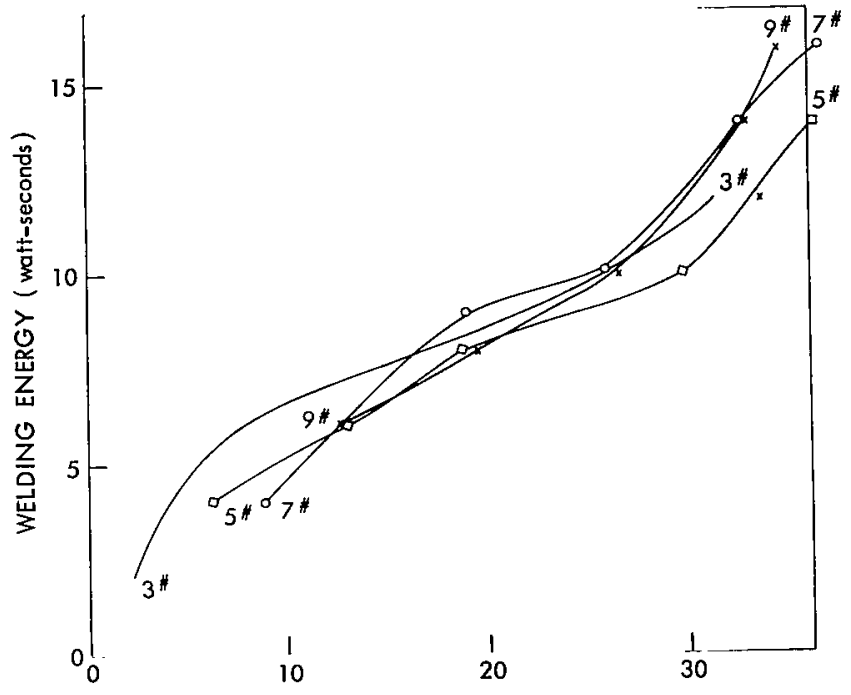

AVERAGE EMBEDMENT (percent of wire diameter)

Figure 36-Average embedment plotted as a function of welding energy for 0.020 inch diameter Alloy 180 welded to 0.012 inch $\times 0.022$ inch Alloy 180 ribbon. 
3. Increased electrode force produces a larger interfacial contact area which, due to the lower resistance, produces less heat in the joint. The end result may be a decreased embedment with an increase in electrode force if the weld energy settings remain unchanged. An opposite effect may be observed at the high electrode forces.

4. Braze type joints generally have lower strengths and poorer reproducibility than solid state bonds or fusion nuggets. The fusion nuggets are generally more reliable since cleanliness becomes less important.

5. Coatings with low melting points such an tin, lead, or solder, have no adverse effect on microcircuitry weld joints if the base metal has good weldability. If sufficient weld energy is used, these coatings are quickly melted and forced out of the interface.

6. Measurements of embedment with micrometers can be useful as an inspection aid after strength-variation-embedment relationships have been established.

Goddard Space Flight Center

National Aeronautics and Space Administration

Greenbelt, Maryland, July 1, 1966

125-25-04-01-51

\section{BIBLIOGRAPHY}

Allen, G., and Wettstein, J., "Microwelding Electronic Modules," Lockheed Missiles and Space Co., Sunnyvale, Calif.

Armstrong, Lloyd D., "Adoption of Welded Electronic Circuits for Missile and Space Vehicles," ASTIA 272642 Space Technology Laboratory, Los Angeles, Calif., March 24, 1961.

Ayer, W., "System Packaging Analysis and Development," Electronic Packaging and Production, May, June, July, 1963.

"Description and Requirements-Resistance Welding (Spot) for Electronic Lead Connections (General)," Ordnance Specification 10272A, Bureau of Naval Weapons, Department of the Navy, June 12, 1962.

"Design and Fabrication of Resistance Welded Electronic Circuit Modules and Assemblies," Ordnance Document 20576A, Bureau of Naval Weapons, Department of the Navy, December 21, 1962.

Heindl, Joseph C., Alberts, Joseph N., and Brock, Bert D., "Resistance Welding of Electronic Components," Metal Progress, June 1962.

"High Density Packaging-Welding," Manufacturing Research Investigation 243.01, Lockheed Aircraft Corporation, Missiles and Space Division, Sunnyvale, Calif., March 1961.

Novak, D., and Hanna, J., "Fabrication Methods and Techniques for Welded Module Production," El-Pac Co., Inc., Morristown, Pa. 
Oswald, A., "The Development of Weld Schedules," Electronic Packaging and Production, January/ February 1962.

"Process Specification for Determination of Weld Schedules for Electronic Assembly," Specification 3-7-F, The Sippican Corp., Marion, Mass.

"Resistance Welding of Leads and Interconnection Media," Polaris Fleet Ballistics Missile, General Spedification for: Ordnance Specification 11889B, Lockheed Missiles and Space Co., Sunnyvale, Calif., for Bureau of Naval Weapons, Department of the Navy.

Sailer, O., "Advantages of Resistance Welding in Component Packaging," Weldmatic Div., Unitek Corp., Monrovia, Calif.

"Three Dimensional Welded Assemblies," Signal Corps Spec. $S C L-7741$, Signal Corps Electronics Command, U. S. Army, January 2, 1964.

"Weldable Leads for Electronic Component Parts," Military Standard 1276, Department of Defense, Washington, D. C., August 14, 1963.

"Welded Electronic Modules," GSFC Specification $W-1$, Systems Integration Branch, Spacecraft Integration and Sounding Rocket Div., Goddard Space Flight Center.

"Welded Module Wiring, Standard For," MSFC Standard 10-M-01618, Marshall Space Flight Center, National Aeronautics and Space Administration, Huntsville, Alabama, April 12, 1962.

"Welding, Spot, Resistance, Non-Structural, Specifications for," MSFC Specification 10-M-01617, Marshall Space Flight Center, National Aeronautics and Space Administration, April 6, 1962.

"Welding, Spot, Small Electronic Assemblies," Engineering Standard 113-900236, Radiation, Incorporated, Melbourne, Florida, October 11, 1961. 
"The aeronautical and space activities of the United States shall be conducted so as to contribute. . . to the expansion of buman knowledge of phenomena in the atmosphere and space. The Administration shall provide for the widest practicable and appropriate dissemination of information concerning its activities and the results thereof."

- National Aeronautics and Space ACt of 1958

\section{NASA SCIENTIFIC AND TECHNICAL PUBLICATIONS}

TECHNICAL REPORTS: Scientific and technical information considered important, complete, and a lasting contribution to existing knowledge.

TECHNICAL NOTES: Information less broad in scope but nevertheless of importance as a contribution to existing knowledge.

TECHNICAL MEMORANDUMS: Information receiving limited distribution because of preliminary data, security classification, or other reasons.

CONTRACTOR REPORTS: Technical information generated in connection with a NASA contract or grant and released under NASA auspices.

TECHNICAL TRANSLATIONS: Information published in a foreign language considered to merit NASA distribution in English.

TECHNICAL REPRINTS: Information derived from NASA activities and initially published in the form of journal articles.

SPECIAL PUBLICATIONS: Information derived from or of value to NASA activities but not necessarily reporting the results of individual NASA-programmed scientific efforts. Publications include conference proceedings, monographs, data compilations, handbooks, sourcebooks, and speçial bibliographies.

Details on the availability of these publications may be obtained from:

SCIENTIFIC AND TECHNICAL INFORMATION DIVISION

NATIONAL AERONAUTICS AND SPACE ADMINISTRATION

Washington, D.C. 20546 